\title{
Narrow-band hard-x-ray lasing with highly charged ions
}

\author{
Chunhai Lyu, Stefano M. Cavaletto $\mathbb{B}^{\bowtie}$, Christoph H. Keitel $@$ \& Zoltán Harman
}

A scheme is put forward to generate fully coherent $x$-ray lasers based on population inversion in highly charged ions, created by fast inner-shell photoionization using broadband $x$-ray free-electronlaser (XFEL) pulses in a laser-produced plasma. Numerical simulations based on the Maxwell-Bloch theory show that one can obtain high-intensity, femtosecond $x$-ray pulses of relative bandwidths $\Delta \omega / \omega=10^{-5}-10^{-7}$, by orders of magnitude narrower than in $\mathrm{x}$-ray free-electron-laser pulses for discrete wavelengths down to the sub-ångström regime. Such $x$-ray lasers can be applicable in the study of $x$-ray quantum optics and metrology, investigating nonlinear interactions between $x$-rays and matter, or in high-precision spectroscopy studies in laboratory astrophysics.

Most X-ray free-electron laser (XFEL) facilities in operation or under construction generate hard-x-ray pulses based on the self-amplified spontaneous-emission (SASE) process. Despite their broad $\left(\Delta \omega / \omega \sim 10^{-3}\right)$ and chaotic spectrum ${ }^{1}$, such high-intensity, transversely coherent SASE x-ray pulses have found diverse applications in physics, chemistry and biology ${ }^{2-4}$. However, current and future investigations of $\mathrm{x}$-ray quantum optics ${ }^{5-7}$, nonlinear $\mathrm{x}$-ray scattering ${ }^{8}$, high-resolution $\mathrm{x}$-ray spectroscopy $\mathrm{y}^{9,10}$, frequency-resolved $\mathrm{x}$-ray scattering ${ }^{11,12}$ and coherent $\mathrm{X}$-ray pump-probe experiments ${ }^{13-15}$ also require a longitudinally coherent $\mathrm{x}$-ray source with a narrower and smooth spectrum. Therefore, considerable efforts have been devoted to generate x-ray pulses with better temporal coherence and lower bandwidth ${ }^{5,16-23}$.

Different seeding schemes have been implemented successfully at XFEL facilities ${ }^{16-20}$. In the hard-X-ray regime, the self-seeding mechanism has reduced the relative bandwidth to the level of $5 \times 10^{-5}$ at photon energies of $8-9 \mathrm{keV}\left(\right.$ ref. $\left.{ }^{17}\right)$. However, at higher energies around $30 \mathrm{keV}$, the predicted relative bandwidth for seeded XFELs is approximately $4 \times 10^{-4}$ (ref. ${ }^{1}$ ). Further reduction of the bandwidth with low-gain XFEL oscillators (XFELOs) has also been proposed. By recirculating the $\mathrm{X}$-ray pulses through an undulator in a cavity, the output $\mathrm{X}$-rays have an estimated relative bandwidth as small as $10^{-7}$ (refs. ${ }^{5,21}$ ). To date, however, the XFELO scheme remains untested.

X-ray lasers (XRLs) adopting atomic transitions provide an alternative approach towards $\mathrm{x}$-ray sources with high brightness and temporal coherence ${ }^{22-28}$. Plasma-based XRLs have achieved saturated amplification for different wavelengths in the soft-X-ray regime. Such soft-XRLs are mainly based on the $3 p \rightarrow 3 s$ or $4 d \rightarrow 4 p$ transitions in $\mathrm{Ne}$ - or Ni-like highly charged ions (HCIs) for elements varying from $\mathrm{Si}$ to $\mathrm{Au}$, where the population inversion is achieved through electron collisional excitation in a hot dense plasma with lengths from a few centimeters up to tens of centimeters ${ }^{26-28}$. By employing a seeding or a different pumping scheme ${ }^{28}$, saturated soft-x-ray lasing from a millimeter-long high-gain plasma has also been realized. The first hard-XRL was initially proposed through direct pumping of the $1 s^{-1} \rightarrow 2 p^{-1}$ transition via photoionization of a K-shell electron ${ }^{29}$. Limited by the high pump power required, this scheme was demonstrated only in recent years after XFELs became available. As a result of the high XFEL-pumping efficiency, an XRL at $846 \mathrm{eV}$ with saturated intensity was achieved in a 2.8-mm-long neon gas ${ }^{22}$, with an estimated fractional bandwidth of $3 \times 10^{-4}$ due to fast Auger decay of the upper lasing state. In an experiment with solid copper, an XRL with a sub-saturation intensity at a photon energy of 8 $\mathrm{keV}$ was produced ${ }^{23}$, with a measured relative bandwidth of $2 \times 10^{-4}$. Reduction of the bandwidth by another order of magnitude via the long-lived $2 p \rightarrow 1 s$ dipole transition in $\mathrm{Ne}^{9+}$ generated by the XFEL pump pulse was also discussed ${ }^{30}$, but it becomes inefficient for heavier ions with transition energies larger than $10 \mathrm{keV}$. For example, the spontaneous emission rate of the $2 p \rightarrow 1 s$ electric-dipole transition, scaling as $Z^{4}$ (with $Z$ the atomic number), is around $1 / 160 \mathrm{fs}^{-1}$ for $\mathrm{Ne}^{9+}$ with a transition energy of $1.022 \mathrm{keV}$, and becomes $1 / 1.7 \mathrm{fs}^{-1} \mathrm{for} \mathrm{Ga}^{30+}$ with a transition energy of $10 \mathrm{keV}$. Alternative schemes to obtain narrow-band XRLs at even higher photon energies, e.g., deep into the hard-x-ray regime, are thus required.

Here we put forward a plasma-based XRL based on the $1 s 2 l \rightarrow 1 s^{2}(l=s, p)$ transition in He-like HCIs and pumped by an XFEL pulse (Fig. 1). The detrimental Auger-decay channel is nonexistent due to the lack of

Max-Planck-Institut für Kernphysik, Saupfercheckweg 1, 69117, Heidelberg, Germany. ${ }^{\circledR e}$-mail: smcavaletto@ gmail.com 


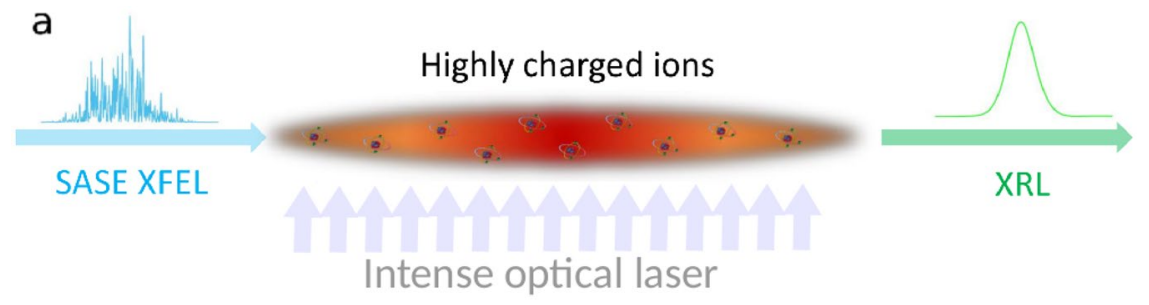

b

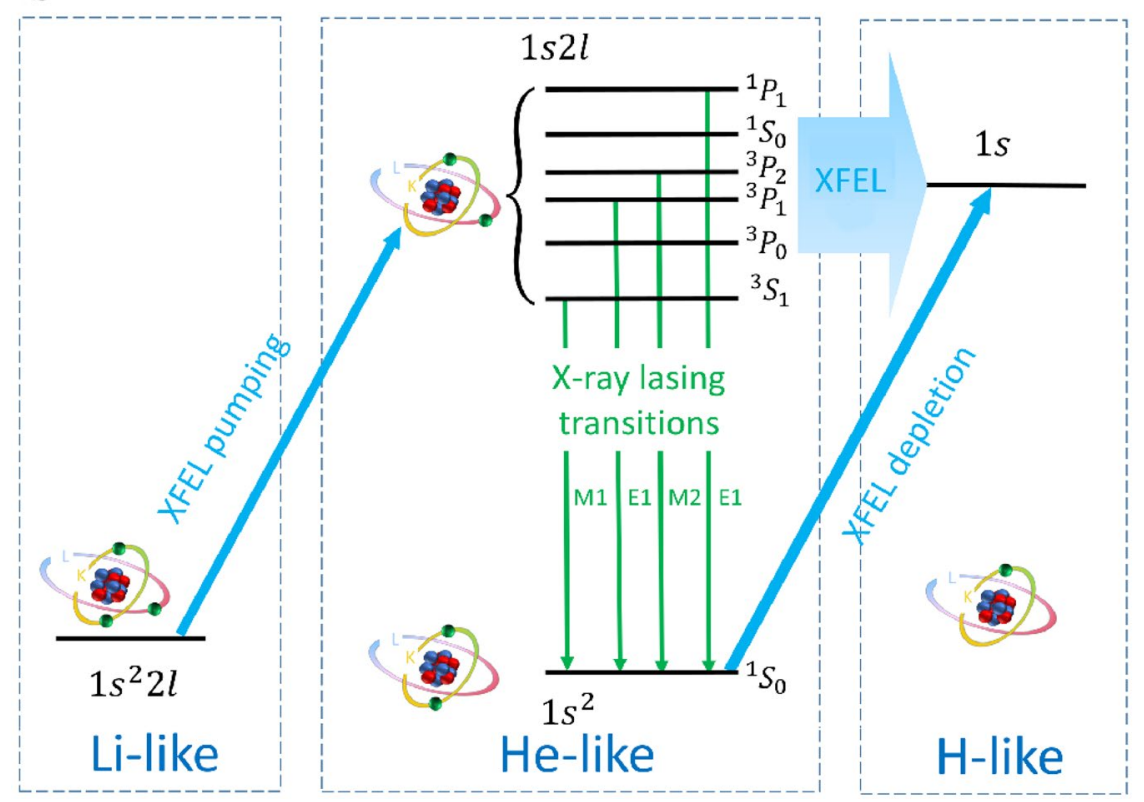

Figure 1. Scheme of the lasing process. (a) Laser configuration. (b) Level scheme. The order of the $1 s 2 l(l=s, p)$ states may vary for different elements (see Table 1). Figure adapted from C. Lyu’s Ph.D. thesis ${ }^{99}$.

\begin{tabular}{|c|c|c|c|c|c|c|c|c|}
\hline \multirow[b]{3}{*}{$1 s 2 l$} & \multicolumn{2}{|l|}{$\mathrm{Ne}^{8+}$} & \multicolumn{2}{|l|}{$A \mathbf{r}^{16+}$} & \multicolumn{2}{|l|}{$\mathbf{K r}^{34+}$} & \multicolumn{2}{|l|}{$\mathrm{Xe}^{52+}$} \\
\hline & $\hbar \omega_{0}$ & $\tau$ & $\hbar \omega_{0}$ & $\tau$ & $\hbar \omega_{0}$ & $\tau$ & $\hbar \omega_{0}$ & $\tau$ \\
\hline & \begin{tabular}{|l|}
$(\mathrm{keV})$ \\
\end{tabular} & (s) & (keV) & (s) & (keV) & (s) & (keV) & (s) \\
\hline${ }^{1} P_{1}: E 1$ & 0.9198 & $\underline{1.08 \times 10^{-13}}$ & 3.137 & $\underline{9.11 \times 10}=-15$ & 13.111 & $6.53 \times 10^{-16}$ & 30.625 & $1.47 \times 10^{-16}$ \\
\hline${ }^{1} S_{0}: \times$ & 0.9134 & $3.14 \times 10^{-04}$ & 3.122 & $6.33 \times 10^{-06}$ & 13.024 & $1.26 \times 10^{-06}$ & 30.210 & $1.66 \times 10^{-07}$ \\
\hline${ }^{3} P_{2}: M 2$ & 0.9125 & $6.24 \times 10^{-09}$ & 3.124 & $1.24 \times 10^{-09}$ & 13.087 & $9.46 \times 10^{-12}$ & 30.589 & $\underline{3.44 \times 10^{-13}}$ \\
\hline${ }^{3} P_{1}: E 1$ & 0.9123 & $1.90 \times 10^{-10}$ & 3.121 & $\underline{5.67 \times 10=13}$ & 13.023 & $2.54 \times 10=-15$ & 30.201 & $3.27 \times 10^{-16}$ \\
\hline${ }^{3} P_{0}: \times$ & 0.9122 & $6.77 \times 10^{-09}$ & 3.120 & $3.41 \times 10^{-09}$ & 13.020 & $1.34 \times 10^{-09}$ & 30.207 & $6.39 \times 10^{-10}$ \\
\hline${ }^{3} S_{1}: M 1$ & 0.9025 & $1.10 \times 10^{-04}$ & 3.101 & $2.30 \times 10^{-07}$ & 12.976 & $1.80 \times 10^{-10}$ & 30.124 & $2.68 \times 10=\frac{12}{-12}$ \\
\hline
\end{tabular}

Table 1. Energy $\hbar \omega_{0}$ and lifetime $\tau$ of the $1 s 2 l$ states in He-like ions. Values are obtained from multiconfiguration Dirac-Hartree-Fock calculations ${ }^{55}$. The $\mathrm{K}_{\alpha}$ transitions from the ${ }^{1} S_{0}$ and ${ }^{3} P_{0}$ states in the $1 s 2 l$ configuration to the ${ }^{1} S_{0}$ state in the $1 s^{2}$ configuration are forbidden by selection rules, and their radiative decay to the other $1 s 2 l$ states is negligibly slow as indicated by the long lifetime. The lifetimes of the lasing transitions, satisfying the condition $1 \mathrm{fs}<\tau<10 \mathrm{ps}$, are underlined.

outer-shell electrons. By choosing a non-dipole lasing transition which decays slowly, this enables saturated lasing for photon energies well above $10 \mathrm{keV}$ and it results in a further reduction of the XRL bandwidth by several orders of magnitude. Such coherent XRLs would enable applications in the growing field of X-ray quantum optics ${ }^{5-7,31-37}$ and metrology $y^{9,10}$, in addition to investigations of nonlinear interactions between $\mathrm{X}$-rays and matter ${ }^{8,38-44}$, and high-precision spectroscopy studies in laboratory astrophysics ${ }^{45,46}$.

\section{Results}

X-ray lasing scheme. The photoionization-pumped atomic laser is illustrated in Fig. 1a. First, a line-focused intense optical laser generates a plasma of highly charged ions, which mainly consists of Li-like ions in a $1 s^{2} 2 l$ state and He-like ions in a $1 s^{2}$ state (see Methods). Then, a SASE XFEL pulse, tuned above the K-edge of He-like ions in the $1 s^{2}$ state, is injected perpendicularly to the optical laser beam. This XFEL pulse immediately removes 


\begin{tabular}{|c|c|c|c|c|c|c|c|}
\hline \multirow[b]{3}{*}{$1 s 2 l$} & \multicolumn{2}{|c|}{$1 s^{2} 2 l \rightarrow 1 s 2 l$} & \multicolumn{2}{|c|}{$1 s^{2} \rightarrow 1 s$} & \multicolumn{2}{|c|}{$1 s 2 l \rightarrow 1 s$} & \multirow{2}{*}{\begin{tabular}{|l}
$\mathbf{s} 2 \mathbf{l} \rightarrow \mathbf{2}$ \\
$\hbar \omega_{\mathrm{T} 2}$ \\
\end{tabular}} \\
\hline & $\hbar \omega_{\mathrm{T} 0}$ & $\sigma_{0}$ & $\hbar \omega_{\mathrm{Tg}}$ & $\sigma_{\mathrm{g}}$ & $\hbar \omega_{\mathrm{Te}}$ & $\sigma_{\mathrm{e}}$ & \\
\hline & $(\mathrm{keV})$ & $(\mathbf{k b})$ & $(\mathrm{keV})$ & $(\mathbf{k b})$ & (keV) & $(\mathbf{k b})$ & $(\mathrm{keV})$ \\
\hline $\mathrm{Ne}^{8+1} P_{1}$ & 1.138 & 39.1 & 1.196 & 151 & 0.274 & 1.55 & 1.295 \\
\hline $\mathrm{Ar}^{16+3} P_{1}$ & 4.013 & 24.6 & 4.124 & 44.6 & 0.998 & 0.48 & 4.312 \\
\hline $\operatorname{Ar}^{16+1} P_{1}$ & 4.029 & 12.4 & 4.124 & 44.6 & 0.982 & 0.47 & 4.312 \\
\hline $\mathrm{Kr}^{34+3} P_{2}$ & 17.048 & 6.24 & 17.315 & 10.9 & 4.206 & 0.12 & 17.635 \\
\hline $\mathrm{Xe}^{52+3} P_{2}$ & 39.949 & 2.66 & 40.302 & 5.31 & 9.671 & 0.06 & 40.961 \\
\hline
\end{tabular}

Table 2. K- and L-shell ionization thresholds $\hbar \omega_{\mathrm{T} 0}, \hbar \omega_{\mathrm{Tg}}, \hbar \omega_{\mathrm{Te}}, \hbar \omega_{\mathrm{T} 2}$ and cross sections $\sigma_{0}, \sigma_{\mathrm{g}}, \sigma_{\mathrm{e}}$. The photoionization cross sections $\sigma_{0}, \sigma_{\mathrm{g}}$ and $\sigma_{\mathrm{e}}\left(\mathrm{kb}=10^{-21} \mathrm{~cm}^{2}\right)$ are calculated with the LANL Atomic Physics $\operatorname{Codes}^{47}$, by assuming a corresponding XFEL photon energy of $\omega_{\text {xfel }}=\omega_{\mathrm{Tg}}$. The cross sections $\sigma_{0}$ and $\sigma_{\mathrm{g}}$ only account for the ionization channel to and from the corresponding upper lasing state in the first column, respectively. Values for other channels can be found in the Supplemental Materials. Due to $\omega_{\mathrm{T} 2}-\omega_{\mathrm{Tg}} \gg \Delta \omega_{\mathrm{xfel}}$ ( $\Delta \omega_{\text {xfel }}$ is the bandwidth of the corresponding XFEL pulse), the single-photon ionization channel $1 s 2 l \rightarrow 2 l$ is energetically forbidden.

a K-shell electron from each of the $\mathrm{Li}$ - and He-like ions, creating He-like ions in the $1 s 2 l$ excited states and $\mathrm{H}$-like ions in the $1 s$ state, respectively (blue arrows in Fig. 1b). Further K-shell ionization from these states is prevented by higher binding energies. Subsequent decay of He-like ions from the $1 s 2 l$ state to the $1 s^{2}$ ground state (green arrows in Fig. 1b) leads to emission of $\mathrm{x}$-ray photons via four possible $\mathrm{K}_{\alpha}$ transitions: one magnetic-dipole (M1) transition from the ${ }^{3} S_{1}$ state, two electric-dipole $(E 1)$ transitions from the ${ }^{3} P_{1}$ or ${ }^{1} P_{1}$ states, and one magnetic-quadrupole (M2) transition from the ${ }^{3} P_{2}$ state. The energy and lifetime of these transitions for suitable XRL systems are shown in Table 1, while Table 2 displays $\mathrm{K}$ - and L-shell ionization thresholds ${ }^{47}$. The ionization-potential depression in the plasma is small and can thus be neglected ${ }^{48}$. The far-detuned L-shell photoionization (broad blue arrow in Fig. 1b) may also depopulate the excited state, but its rate is much slower compared to the pump rates. Thus, the slow decay of these excited states leads to a transient population inversion in the He-like ions, and to the amplification of the emitted $\mathrm{x}$-rays, i.e., transient $\mathrm{x}$-ray lasing.

Lasing transitions. Two factors determine which transition will lase. First, typical XFEL facilities operate at peak photon fluxes of $10^{33}-10^{35} \mathrm{ph} . / \mathrm{cm}^{2} / \mathrm{s}$ (refs. ${ }^{1,4}$ ). For the photoionization cross sections shown in Table 2 $\left(\right.$ ref. ${ }^{47}$ ), they yield inverse ionization rates of a few femtoseconds. Transitions with lifetimes longer than $1 \mathrm{fs}$ are thus necessary to ensure population inversion. Second, for the ions we are going to consider, the hydrodynamic plasma expansion time of $\sim 10$ ps (refs. ${ }^{49,50}$ ), depending on the plasma size and temperature, also influences the lasing process. Sufficient $\mathrm{x}$-ray amplification takes place only from transitions whose upper-state lifetimes satisfy $1 \mathrm{fs}<\tau<10 \mathrm{ps}$. Systems and transitions satisfying this condition are underlined in Table 1 . Although current XFEL facilities are designed to deliver $\mathrm{x}$-rays with a single-photon energy up to $30 \mathrm{keV}$ (ref. ${ }^{1}$ ), calculations for $\mathrm{Xe}^{52+}$ requiring 40.3-keV FEL frequencies were included as an example of the applications which would be enabled by such XFEL pulses in the near future ${ }^{4}$.

For light ions, $\mathrm{Ne}^{8+}$ for example, the $E 1$ transition with a decay rate of $9.2 \times 10^{12} \mathrm{~s}^{-1}$ from the ${ }^{1} P_{1}$ state will develop lasing. The other transitions have decay times much larger than the plasma expansion time, and their contribution is negligible compared to the ${ }^{1} P_{1}$ state. For heavy ions such as $\mathrm{Xe}^{52+}$, however, the two $E 1$ transition rates scale as $\sim Z^{4}$ ( $Z$ being the atomic number), corresponding to $3.0 \times 10^{15} \mathrm{~s}^{-1}$ for ${ }^{3} P_{1}$ and $6.8 \times 10^{15} \mathrm{~s}^{-1}$ for ${ }^{1} P_{1}$, respectively, which are too large to enable population inversion with available XFEL pulses. On the other hand, the decay rates of the $M 1$ transition from the ${ }^{3} S_{1}$ state and of the $M 2$ transition from the ${ }^{3} P_{2}$ state are $3.7 \times 10^{11} \mathrm{~s}^{-1}$ and $2.6 \times 10^{12} \mathrm{~s}^{-1}$, respectively, which is sufficient for lasing to take place before the expansion of the plasma.

Two-level approximation. Since the lifetimes of different $\mathrm{K}_{\alpha}$ transitions from the $1 s 2 l$ configuration differ by orders of magnitude, when we model the lasing process from a specific transition, a two-level approximation for the He-like ions is applicable. This is evident for the case of $\mathrm{Ne}^{8+}$ ions, where only the $E 1$ transition from the ${ }^{1} P_{1}$ state satisfies the requirements for lasing described above. For $\mathrm{Ar}^{16+}$ ions, both $E 1$ transitions may lase simultaneously. Thus, the XFEL photon energy $\omega_{\text {xfel }}$ and the mean peak flux have to be tuned properly, such that when the transition from the ${ }^{3} P_{1}$ state is pumped, the other transition does not lase due to the relatively fast decay rate of the ${ }^{1} P_{1}$ state. On the other hand, when lasing from the ${ }^{1} P_{1}$ state is considered, the effects from the ${ }^{3} P_{1}$ state can be neglected as well due to its slow lasing dynamics. Similar approximations can be applied when we model the XRLs from $\mathrm{Kr}^{34+}$ and $\mathrm{Xe}^{52+}$ ions which also contain two suitable lasing transitions. Furthermore, for the plasma conditions considered later, collision-induced population transfer rates between different upper states are small compared to the $\mathrm{K}_{\alpha}$ emission rate, ensuring the validity of the two-level approximation.

Initial charge-state distributions. Our scheme requires a plasma in which the density of Li-like ions is maximized compared to other ionic species. While the generation of such a plasma, in the initial stage (of a few femtoseconds), involves highly complex and nonlinear laser-matter interaction processes for the ejection of outermost electrons, the removal of deeply bounded electrons in the later stage of the plasma generation is dominated by linear processes such as collisional ionization. Therefore, when we focus on the state of the plasma after the HCIs are generated, collisional-radiative atomic-physics plasma codes such as FLYCHK ${ }^{51}$ can be used for an estimation of the properties of the plasma, such as the ion density $N_{\text {ion }}$ and the electron temperature $T_{\mathrm{e}}$ necessary 


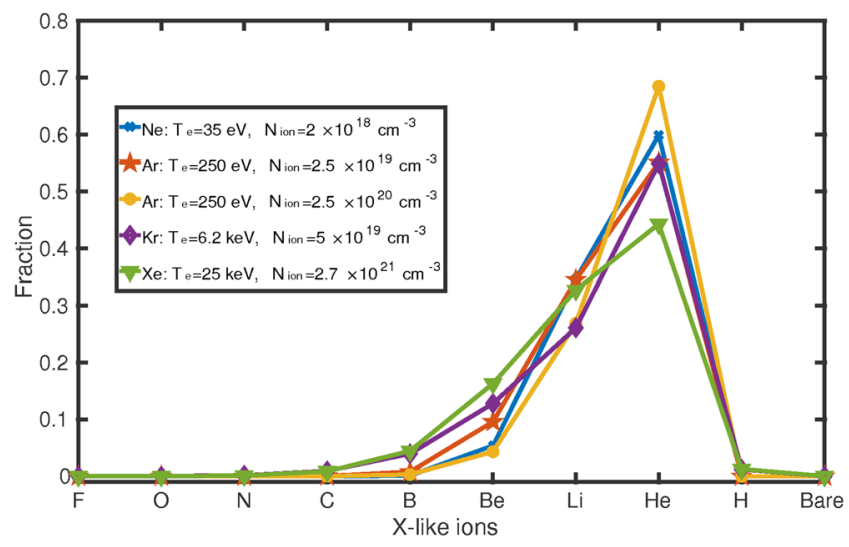

Figure 2. Charge-state distributions in laser-produced plasmas. Electron temperature $T_{\mathrm{e}}$ and ion density $N_{\text {ion }}$ are chosen to enable a significant fraction of Li-like ions in the plasma ${ }^{51}$. For the Ar plasma, the red (yellow) line with $N_{\text {ion }}=2.5 \times 10^{19} \mathrm{~cm}^{-3}\left(N_{\text {ion }}=2.5 \times 10^{20} \mathrm{~cm}^{-3}\right)$ is tuned to achieve optimal lasing from the ${ }^{3} P_{1}\left({ }^{1} P_{1}\right)$ state. Figure reproduced from C. Lyu's Ph.D. thesis ${ }^{99}$.

\begin{tabular}{|c|c|c|c|c|c|c|c|c|c|c|c|c|}
\hline \multicolumn{3}{|l|}{ Transitions } & \multicolumn{3}{|c|}{ Line broadenings } & \multicolumn{4}{|l|}{ XFELs } & \multicolumn{3}{|c|}{ Simulation results } \\
\hline Upper state & $\hbar \omega_{0}$ & $\hbar \Gamma$ & $\hbar \Delta \omega_{\mathrm{D}}$ & $\hbar \Delta \omega_{\mathrm{e}-\mathrm{i}}$ & $\hbar \Delta \omega_{\mathrm{i}-\mathrm{i}}$ & peak flux & $\Delta t_{\mathrm{xfel}}$ & $\hbar \Delta \omega_{\mathrm{xfel}}$ & $S_{\text {focal }}$ & $L_{\mathrm{c}}$ & $I_{c}$ & \\
\hline $1 s 2 l$ & $(\mathrm{keV})$ & $(\mathrm{meV})$ & $(\mathrm{meV})$ & $(\mathrm{meV})$ & $(\mathrm{meV})$ & $\left(\mathrm{ph} . / \mathrm{cm}^{2} / \mathrm{s}\right)$ & (fs) & $(\mathrm{eV})$ & $\left(\mu \mathbf{m}^{2}\right)$ & $(\mathrm{mm})$ & $\left(\mathrm{W} / \mathrm{cm}^{2}\right)$ & $\Delta \omega / \omega$ \\
\hline $\mathrm{Ne}^{8+1} P_{1}$ & 0.920 & 6.08 & 3.23 & 0.08 & 0.06 & $1.1 \times 10^{34}$ & 21.3 & 6.25 & 1.5 & 2.8 & $4.5_{-4.4}^{+9.3} \times 10^{12}$ & $7.8_{-2.7}^{+4.3} \times 10^{-5}$ \\
\hline $\operatorname{Ar}^{16+3} P_{1}$ & 3.121 & 1.16 & 7.80 & 1.38 & 9.81 & $2.3 \times 10^{33}$ & 124 & 1.55 & 1.0 & 3.3 & $5.0_{-4.0}^{+3.8} \times 10^{14}$ & $7.8_{-2.1}^{+1.9} \times 10^{-6}$ \\
\hline $\operatorname{Ar}^{16+1} P_{1}$ & 3.137 & 72.2 & 7.83 & 2.20 & 25.22 & $2.0 \times 10^{35}$ & 2.0 & 74 & 0.1 & 0.35 & $1.5_{-0.8}^{+1.4} \times 10^{16}$ & $4.7_{-1.7}^{+2.0} \times 10^{-5}$ \\
\hline
\end{tabular}

Table 3. XRL transitions in He-like Ne and Ar ions. For the XFEL peak flux, ph. stands for photons. The uncertainties in the simulation results for $I_{\mathrm{c}}$ and $\Delta \omega / \omega$ arise from the random XFEL pulse profiles and the noisy spontaneous-emission seedings, with the upper and lower bounds being the values at the 10th and 90th percentiles of the corresponding distributions [as shown in Fig. 3(d,f) for $\mathrm{Ar}^{16+3} P_{1}$ ]. Table adapted from C. Lyu's Ph.D. thesis ${ }^{99}$.

to satisfy the corresponding ionic distributions. Then, the pondermotive scaling law can be used to estimate the laser intensity and wavelength needed to produce such a plasma (see Discussions). The charge-state distributions obtained from such simulations are displayed in Fig. 2, where ions with other charge states coexist with the Li-like ions. We notice in particular that most of the ions are He-like ions in the $1 s^{2}$ state with negligible populations in the $1 s 2 l$ state. This is due to the fact that, even though energetic electrons can impact with the ions of any charge state and create $\mathrm{K}$-shell holes, these are immediately filled by the outer-shell electrons, leading to the emission of incoherent $\mathrm{K}_{\alpha}$ photons in the hard-x-ray regime ${ }^{52-54}$.

Electron-impact K-shell ionization or excitation are very slow compared to the spontaneous decay of the excited state, such that population inversion does not set in and spontaneous $\mathrm{K}_{\alpha}$ radiation is not amplified. However, when an intense XFEL pulse is injected, the K-shell photoionization rates of the Li-like ions can be much larger than the decay rate of the $1 s 2 l$ excited state of the resulting He-like ions. Since the lower lasing state $\left(1 s^{2}\right)$ of the He-like ions is also depleted by the XFEL pulse with a large photoionization rate, population inversion in the He-like ions will be reached shortly after the arrival of the XFEL pulse.

XRLs from He-like $\mathrm{Ne}$ and Ar. Parameters for x-ray lasing from transitions in He-like $\mathrm{Ne}$ and Ar ions are shown in Table 3. The natural linewidth $\Gamma=1 / \tau$ for each lasing transition is obtained from multiconfiguration Dirac-Hartree-Fock calculations ${ }^{55}$. Doppler broadening $\Delta \omega_{\mathrm{D}}$, electron-ion impact broadening $\Delta \omega_{\mathrm{e}-\mathrm{i}}$, and ionion Stark broadening $\Delta \omega_{\mathrm{i}-\mathrm{i}}$, are calculated for given $T_{\mathrm{e}}$ and $N_{\text {ion }}$ as shown in Fig. 2 based on Maxwell-Boltzmann distributions ${ }^{56}$, with ion temperature $T_{\mathrm{i}}=500 \mathrm{~K}$ (see Methods). XFEL parameters such as peak flux and pulse duration $\Delta t_{\mathrm{xfel}} \approx 0.2 \tau$ are selected in order to ensure photoionization rates larger than the decay rate of the $1 \mathrm{~s} 2 \mathrm{l}$ excited state in He-like ions, and the onset of population inversion. The XFEL spectral width $\Delta \omega_{\text {xfel }}$ is set by assuming an XFEL coherence time of $0.01 \tau$ for the sake of computational cost. Though such an assumption may result in $\Delta \omega_{\text {xfel }} / \omega_{\text {xfel }} \ll 0.5 \%$, see, e.g., Table 4 for $\mathrm{Kr}$ and Xe ions, it does not modify the pumping efficiency as the photoionization cross section varies slowly within the pulse bandwidth, which is $1 \%$ of the central photon energy. The focal size $S_{\text {focal }}$ of the XFEL pulse is chosen to ensure the required peak flux for the x-ray photon energy listed in Table 3, as well as to ensure that the divergence of the XFEL beam is negligible during its propagation through the plasma (see Methods).

We simulate the lasing process by solving the Maxwell-Bloch equations ${ }^{57}$ numerically in retarded-time coordinates for 1,000 different realizations of SASE XFEL pulses. The propagation and absorption of the XFEL pulses 


\begin{tabular}{|c|c|c|c|c|c|c|c|c|c|c|c|c|}
\hline \multicolumn{3}{|l|}{ Transitions } & \multicolumn{3}{|c|}{ Line broadenings } & \multicolumn{4}{|l|}{ XFELs } & \multicolumn{3}{|c|}{ Simulation results } \\
\hline Upper state & $\hbar \omega_{0}$ & $\hbar \Gamma$ & $\hbar \Delta \omega_{\mathrm{D}}$ & $\hbar \Delta \omega_{\mathrm{e}-\mathrm{i}}$ & $\hbar \Delta \omega_{\mathrm{i}-\mathrm{i}}$ & peak flux & $\Delta t_{\text {xfel }}$ & $\hbar \Delta \omega_{\mathrm{xfe}}$ & $S_{\text {focal }}$ & $L_{\mathrm{c}}$ & $I_{\mathrm{c}}$ & \\
\hline $1 s 2 l$ & $(\mathrm{keV})$ & $(\mathrm{meV})$ & $(\mathrm{meV})$ & $(\mathrm{meV})$ & $(\mathrm{meV})$ & $\left(\mathrm{ph} . / \mathrm{cm}^{2} / \mathrm{s}\right)$ & (fs) & $(\mathrm{eV})$ & $\left(\mu \mathbf{m}^{2}\right)$ & $(\mathrm{mm})$ & $\left(\mathrm{W} / \mathrm{cm}^{2}\right)$ & $\Delta \omega / \omega$ \\
\hline $\mathrm{Kr}^{34+3} P_{2}$ & 13.087 & 0.07 & 22.58 & 0.45 & 2.76 & $1.9 \times 10^{34}$ & 202 & 0.57 & 0.10 & 289 & $4.3_{-4.2}^{+7.6} \times 10^{16}$ & $3.7_{-1.1}^{+1.6} \times 10^{-7}$ \\
\hline $\mathrm{Xe}^{52+3} P_{2}$ & 30.589 & 1.91 & 42.16 & 7.60 & 126 & $7.4 \times 10^{34}$ & 72.5 & 1.96 & 0.13 & 8.5 & $4.7_{-4.5}^{+6.9} \times 10^{18}$ & $1.5_{-0.5}^{+0.6} \times 10^{-6}$ \\
\hline
\end{tabular}

Table 4. Same as Table 3 for He-like Kr and Xe ions. The values of the bandwidths $\Delta \omega_{\text {xfel }}$ are relatively smaller than the realistic values at XFEL facilities (see Methods), and are used for the sake of computational cost. Using realistic bandwidths does not modify the conclusions as long as the photoionization cross section changes slowly within the realistic frequency bands. Therefore, when we compare the bandwidth of the XRLs and XFEL pulses, we refer to the realistic bandwidth measured at XFEL facilities and not to the values used in this table. Table adapted from C. Lyu's Ph.D. thesis ${ }^{99}$.

are accounted for through rate equations describing the evolution of the corresponding photon flux, while the $\mathrm{XRL}$ is modeled as an electromagnetic field in resonance with the corresponding lasing transition of the He-like ions. The results in Table 3 present the XRL intensity $I_{\mathrm{c}}$ and relative bandwidth $\Delta \omega / \omega$ at the characteristic plasma length $L_{\mathrm{c}}$, with $L_{\mathrm{c}}$ being the optimal length (as defined in Fig. 3 ) to obtain high-intensity x-ray pulses with narrow bandwidth. All the transitions are predicted to generate high-intensity $\mathrm{x}$-ray pulses for plasma lengths around 3

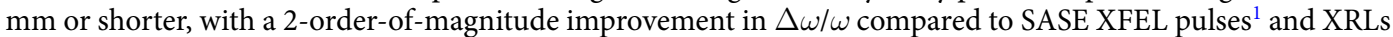
with neutral atoms $\mathrm{s}^{22,23}$.

Evolution of the XRLs. To understand the properties of our XRL and how it develops in the plasma, simulation results for the ${ }^{3} P_{1} \rightarrow{ }^{1} S_{0}$ transition in $\mathrm{Ar}^{16+}$ are shown in Figs. 3 and 4 . We use a partial-coherence method $^{58}$ to simulate 124-fs-long SASE XFEL pulses with a spectral width of $\hbar \Delta \omega_{\text {xfel }}=1.55 \mathrm{eV}$ and peak photon flux of $2.3 \times 10^{33} \mathrm{~cm}^{-2} \mathrm{~s}^{-1}$. This results in a peak pumping rate of $5.6 \times 10^{13} \mathrm{~s}^{-1}$ for the upper lasing state, and a depletion rate of $1.0 \times 10^{14} \mathrm{~s}^{-1}$ for the lower lasing state. They are 32 and 58 times larger than the spontaneous-emission rate of the ${ }^{3} P_{1}$ state, ensuring population inversion.

Averaged results over 1,000 SASE-pulse realizations are shown in Fig. 3a-c. The peak intensity of the XRL, shown by the solid line in Fig. 3a, increases exponentially during the initial propagation stage, then displays a saturation behavior. The dotted line indicates the saturation intensity $I_{s}=\hbar \Gamma \omega_{0}^{3} / 6 \pi c^{2}$ at which the stimulated-emission rate equals the spontaneous-emission rate. This is also the intensity from which the amplification begins to slow down. The evolution of the pulse duration and the spectral width are shown by the solid lines in Fig. 3b,c. At $L=0$, only spontaneous emission takes place: the 342 -fs average pulse duration is mainly determined by the lifetime of the ${ }^{3} P_{1}$ state (Fig. 3b), whereas the $23.5-\mathrm{meV}$ intrinsic spectral width before propagation (Fig. $3 \mathrm{c}$ ) is mostly due to the sum of the natural linewidth $\Gamma$ and the three broadening effects shown in Table 3.

During its propagation in the medium, gain narrowing and saturation rebroadening will also contribute to the final bandwidth. This can be observed by inspecting the four distinct propagation regions separated by $L_{1}, L_{2}$ and $L_{3}$ in Fig. $3 \mathrm{a}-\mathrm{c}$, which can also be followed in Fig. $4 \mathrm{a}$,b for a single simulation. Up to $L_{1}=0.75 \mathrm{~mm}$, both the pulse duration and spectral FWHM decrease severely. The laser intensity and spectrum in this region for a single simulation are spiky and noisy, as the ions irradiate randomly in time and space (Fig. 4a,b). When the spontaneously emitted signal propagates and stimulated emission sets in, it selectively amplifies the frequencies around $\omega_{0}$ such that the XRL pulse approaches a fully coherent transform-limited profile at $L_{1}\left(\right.$ ref. $\left.{ }^{59}\right)$, with a bandwidth smaller than the intrinsic width. Thereafter, a gradual broadening of the spectrum is observed in the region $L_{1} L_{2}$. The broadening increases abruptly from $L_{2}=2.3 \mathrm{~mm}$, where the saturation intensity has been reached and the stimulated-emission rate exceeds the spontaneous-emission rate. This is accompanied by a substantial slowing down of the amplification of the intensity and a significant decrease of the pulse duration in the region between $L_{2}$ and $L_{3}$ (Fig. 3a,b). Further propagation of the XRL pulse after $L_{3}=3.5 \mathrm{~mm}$ is characterized by the onset of Rabi flopping (Fig. 4a) which is reflected by a splitting in the XRL spectrum (Fig. 4b). This effect is much stronger and more marked than for previous XFEL-pumped transient lasers with neutral atoms ${ }^{59}$ due to the absence of Auger decay $^{58}$. At the same time, the gain of the laser intensity in this region is strongly suppressed.

Optimal plasma length. The optimal choice for a coherent XRL pulse is located in the third region $L_{2} L_{3}$, where saturation has already been reached while the bandwidth is still narrow. By choosing the medium length to be $L_{\mathrm{c}}=3.3 \mathrm{~mm}$, as shown in Table 3 and Fig. 3, one will obtain an approximately 87-fs-long XRL pulse with an average peak intensity of $I_{\mathrm{c}}=5.0 \times 10^{14} \mathrm{~W} \mathrm{~cm}^{-2}(\sim 80 \%$ fluctuations) and an average bandwidth of $\hbar \Delta \omega=24.5$ $\mathrm{meV}$ ( $\sim 30 \%$ fluctuations). This can also be seen in Fig. 4(c,d) which show four single-shot XRL pulse and spectrum profiles at length $L_{c}$ for the different realizations of SASE XFEL pulses. On average, the XRL from He-like Ar ions gives $\Delta \omega / \omega=7.8 \times 10^{-6}$ for a total of $8.7 \times 10^{8}$ coherent photons, with a peak brightness of $8.1 \times 10^{30} \mathrm{brt}(\mathrm{brt}$ $=$ photons $/ \mathrm{s} / \mathrm{mm}^{2} / \mathrm{mrad}^{2} / 0.1 \%$ bandwidth is the units of the brightness). Feasibility of our narrowband lasing scheme with the inclusion of plasma inhomogeneities is discussed in the Supplemental Material.

XRLs from He-like $\mathrm{Kr}$ and $\mathrm{Xe.}$. In the light of the promising results obtained for $\mathrm{Ne}$ and $\mathrm{Ar}$, we use our model to predict XRL for more highly charged ions as well. For $\mathrm{Kr}^{34+}$ and $\mathrm{Xe}^{52+}$ ions, the $\mathrm{M} 2$ transitions provide an even more significant reduction of the bandwidth, with $\Delta \omega / \omega$ being $3 \times 10^{-7}$ and $1.5 \times 10^{-6}$, respectively. The resulting 13- and 30-keV lasers feature similar bandwidths as the untested XFELO scheme ${ }^{21}$, with intensities of 

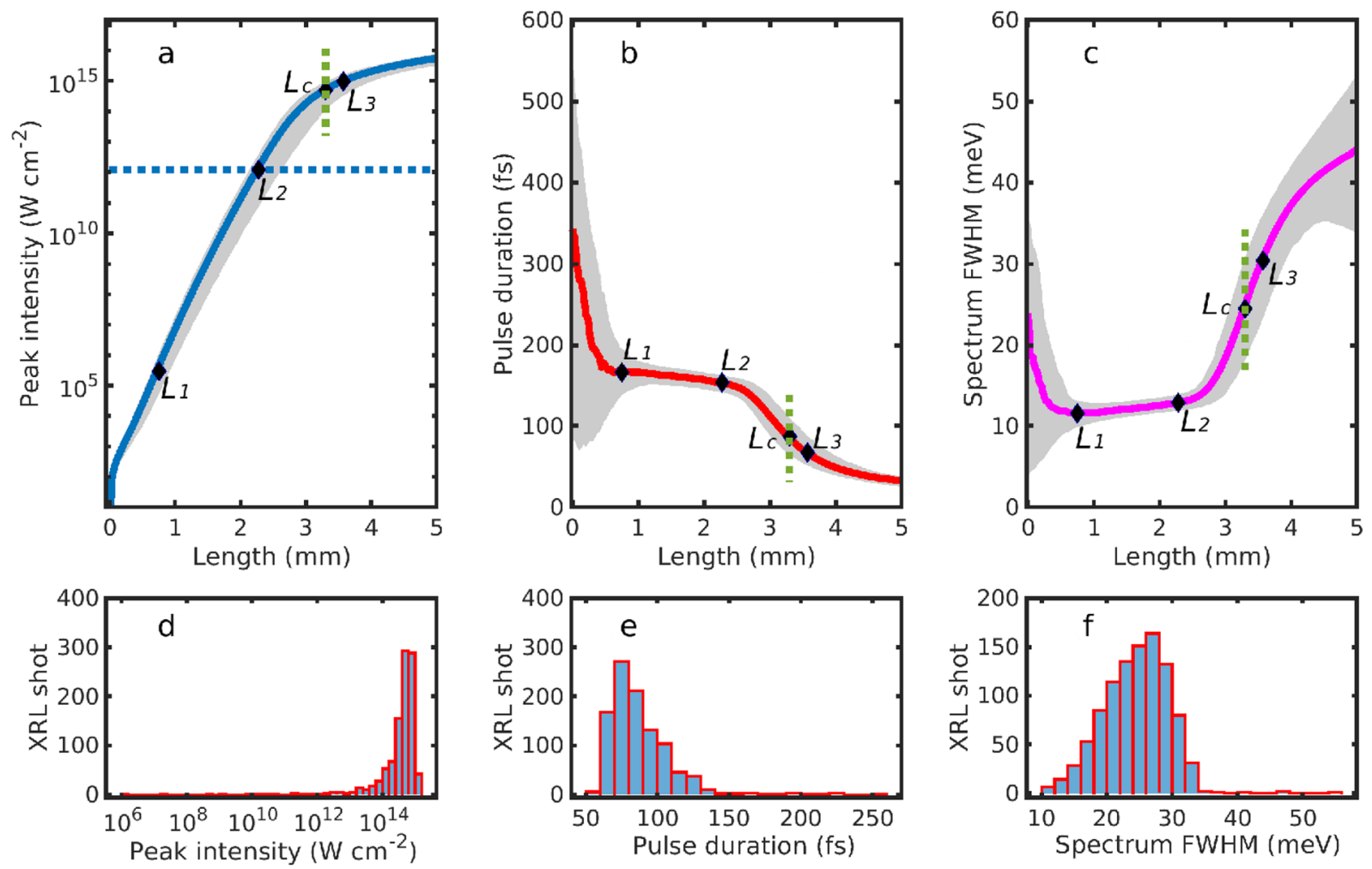

Figure 3. Evolution of the XRLs over 1,000 simulations $\left(\operatorname{Ar}^{16+3} P_{1}\right) .(\mathbf{a}-\mathbf{c})$ Peak intensity, pulse duration and spectral full width at half maximum (FWHM) for the $\mathrm{x}$-ray laser. The solid lines display results averaged over 1,000 simulations. The dotted line in (a) indicates the saturation intensity $I_{\mathrm{s}}=1.18 \times 10^{12} \mathrm{~W} \mathrm{~cm}^{-2} . L_{1}, L_{2}$ and $L_{3}$ mark the lengths at which the XRL pulse reaches transform-limited profile, saturation intensity and Rabi flopping, respectively. $L_{\mathrm{c}}$ refers to the characteristic length at which the slope of the solid line in (a) is $1 / 3$ of the slope at $L_{2}$. The gray areas in $(\mathbf{a}-\mathbf{c})$ indicate the distribution areas of the results over 1,000 simulations. At a given length, the bottom and top edges of the areas indicate the 10th and 90th percentiles of the distributions, respectively. (d-f) Distributions of the peak intensity, pulse duration and spectral FWHM at $L_{\mathrm{c}}$ along the green dotted lines in $(\mathbf{a}-\mathbf{c})$. Figure reproduced from C. Lyu's Ph.D. thesis ${ }^{99}$.

$\sim 10^{18} \mathrm{~W} \mathrm{~cm} \mathrm{~cm}^{-2}$. The relative bandwidths are by 2 to 3 orders of magnitude narrower than the value predicted for future seeded-XFEL sources at analogous hard-X-ray wavelengths around $0.41-0.95 \AA$ ( ref. $^{1}$ ). The experimental generation of such highly charged ions would be challenging ${ }^{53}$, albeit in principle possible via currently available petawatt laser facilities ${ }^{60}$.

Due to large broadening effects, the effective gain coefficient for the XRL from the $M 2$ transition in $\mathrm{Kr}^{34+}$ ions is small, leading to an optimal plasma length of $29 \mathrm{~cm}$. Though such a plasma can be generated with an intense optical laser, diffraction of the XFEL pump beam at long propagation lengths may result in a reduction of the pump rate and an unsaturated XRL (see Methods). A similar low-gain behavior is also observed for the XRLs from the ${ }^{3} P_{1} \rightarrow{ }^{1} S_{0}$ transition in $\mathrm{Kr}^{34+}$ due to fast decay, and for the XRLs from the ${ }^{3} S_{1} \rightarrow{ }^{1} S_{0}$ transition in Xe ${ }^{52+}$ due to large broadening. These two XRLs are thus excluded from Table 4.

Brightness and spatial coherence. The brightness is defined as the number of photons emitted per second within the $0.1 \%$ relative bandwidth under an emittance of $1 \mathrm{~mm} \mathrm{mrad}$ and is calculated through ${ }^{61}$

$$
B[\text { brt }]=\frac{10^{17}}{1.6}\left(\frac{I_{c}}{\hbar \Delta \omega}\right)\left(\frac{S_{\text {focal }}}{\lambda^{2}}\right),
$$

where the units for the intensity $I_{c}$, the bandwidth $\hbar \Delta \omega$, the focal size $S_{\text {focal }}$ on the right hand side of Eq. (1) are the same as shown in Tables 3 and 4, with $\lambda$ being the wavelength in units of nm. Since the Fresnel number is $S_{\text {focal }} / L_{\mathrm{c}} \lambda \lesssim 1$ for our system, the outgoing XRL is transversely coherent, thus it can be described as a Gaussian beam, with $\lambda^{2} / S_{\text {focal }}$, in units of $\mathrm{mrad}^{2}$, being the solid angle the XRL emits into.

For XRLs from light elements, the calculated brightnesses, $3.2 \times 10^{27}$ brt for $\mathrm{Ne}^{8+}{ }^{1} P_{1}, 8.1 \times 10^{30}$ brt for $\mathrm{Ar}^{16+}$ ${ }^{3} P_{1}$ and $4.0 \times 10^{30}$ brt for $\mathrm{Ar}^{16+1} P_{1}$, are smaller than those in XFELs at the same photon energies. For XRLs from heavier elements such as $\mathrm{Kr}$ and Xe, however, with $B=6.1 \times 10^{33}$ and $5.0 \times 10^{35}$ brt, respectively, the brightnesses are significantly increased and can be larger than those available at XFELs, representing a significant advantage of our scheme in generating high-intensity fully coherent XRLs in the hard-x-ray regime. 

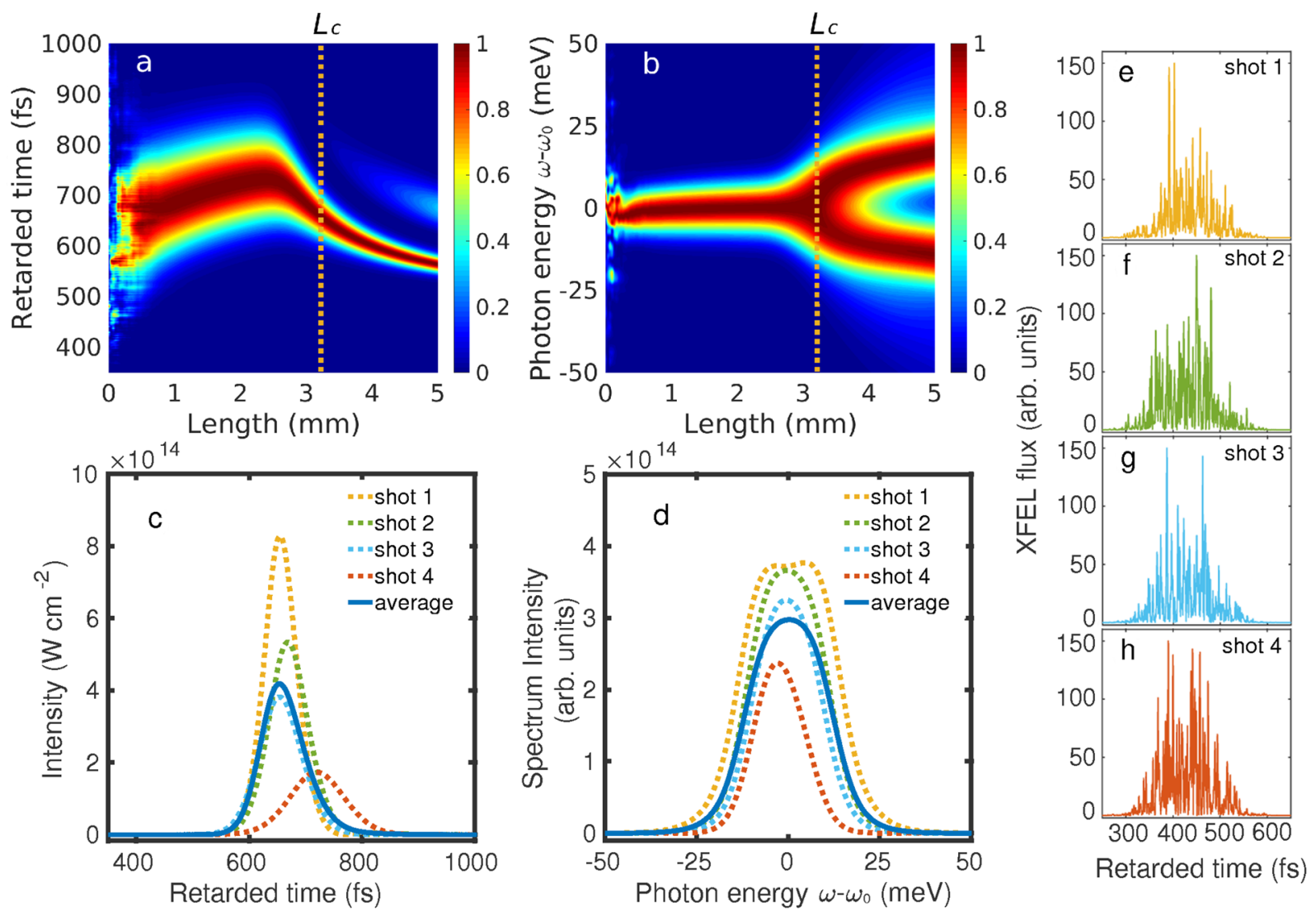

Figure 4. Pulse and spectrum profiles of a single-shot XRL $\left(\operatorname{Ar}^{16+3} P_{1}\right)$. (a,b) Evolution of normalized XRL intensity and power spectrum as a function of plasma length. Color bars: at each plasma length, the temporal and spectral intensities are normalized to their peak values at that length. The strength of the second peak appearing for the intensity profile at large plasma lengths in a has been multiplied by a factor of 5 for better visibility. (c,d) XRL pulse profile and spectrum at $L_{c}$ for the different realizations of SASE XFEL pulses shown in $(\mathbf{e}-\mathbf{h})$. The yellow dotted lines, corresponding to the results from the simulation in (a,b), are associated with shot 1 in (e). The evolution of XRL intensity and spectrum for the XFEL shots $2-4$ in (f-h), and averaged over 1,000 simulations (blue solid lines), can be found in the Supplemental Material. Figure reproduced from C. Lyu's Ph.D. thesis ${ }^{99}$.

The XRL photon fluxes can be calculated from Tables 3 and 4 as $J_{c}=I_{c} / \hbar \omega_{0}$. In units of $\mathrm{ph} . / \mathrm{s} / \mathrm{cm}^{2}$, this gives $J_{c}=3.0 \times 10^{28}, 1.0 \times 10^{30}$ and $3.0 \times 10^{31}$ for the three transitions in Table 3 , respectively, indicating a relatively small conversion efficiency of $\mathrm{x}$-ray photons from the XFEL pulses. However, with $J_{c}=2.1 \times 10^{31}$ and $9.7 \times 10^{32}$ for the two transitions in Table 4, respectively, a significantly larger efficiency is achieved for harder x-ray lasers.

\section{Discussion}

The plasma used in our lasing scheme can be generated by illuminating a target with an intense optical laser ${ }^{49,50}$ : first, the outer-shell electrons of the atoms in the gas undergo tunneling ionization in the presence of the laser; afterwards, their acceleration in the strong laser field and subsequent recollisions with the ions lead to the further ejection of more tightly bound electrons and the formation of dense highly charged ions ${ }^{52-54}$. The critical density and skin effect during the generation of dense plasmas can be overcome by either using optical lasers with shorter wavelengths or by adoption of plasma guiding schemes (see Supplemental Sec. C for additional details).

While an accurate prediction of the laser conditions for the production of the plasmas in Fig. 2 would require simulations involving many nonlinear light-matter interactions going beyond the scope of this article, here we provide a conservative estimation of the laser intensity, pulse duration and total power needed for plasma generation based on the FLYCHK atomic-physics plasma model and verified scaling laws ${ }^{54}$. FLYCHK is first used to obtain the electron temperature $T_{\mathrm{e}}$ required to maximize the density of $\mathrm{Li}$-like ions in the plasma. For such values of $T_{\mathrm{e}}$, the required laser intensity at a given optical wavelength is estimated through the pondermotive scaling $\operatorname{law}^{62,63}$

$$
T_{\mathrm{e}} / \mathrm{keV} \approx 3.6 \times I_{16} \lambda_{\mu}^{2},
$$

where $I_{16}$ is the laser intensity normalized to $1 \times 10^{16} \mathrm{~W} \mathrm{~cm}^{-2}$, and $\lambda_{\mu}$ is the laser wavelength in units of $\mu \mathrm{m}$.

Nanoplasmas consisting of $\mathrm{Ar}^{16+}$ have been obtained from Ar clusters interacting with optical lasers ${ }^{64}$. Incoherent $\mathrm{K}_{\alpha}$ emission from plasmas of He-like Ar ions, with a size varying from 20 to $35 \mathrm{~nm}$, has been recorded 
for laser durations and intensities from $30 \mathrm{fs}$ to $3.5 \mathrm{ps}$ and $10^{17} \mathrm{~W} \mathrm{~cm} \mathrm{~cm}^{-2}$ to $10^{14} \mathrm{~W} \mathrm{~cm}^{-2}$, respectively ${ }^{64}$. To achieve the plasma conditions discussed in Fig. 2, a 2-picosecond-long, 0.44-TW, 800-nm optical laser with an intensity of $1.1 \times 10^{15} \mathrm{~W} \mathrm{~cm}^{-2}$ and a total energy of $0.88 \mathrm{~J}$ per pulse is required (see Methods). High-repetition rate (up to $500 \mathrm{~Hz})$, high-power $(0.3 \mathrm{TW})$ picosecond lasers, with a single-pulse energy up to $1.5 \mathrm{~J}$, have been used for the generation of table-top soft-XRLs ${ }^{65,66}$. Combining such laser systems with XFELs can produce high-repetition rate, high-intensity, narrow-band hard-XRLs with extended applications. The generation of plasmas consisting of highly charged Ne ions would be even less demanding, and could be achieved as well.

Though highly charged $\mathrm{Kr}^{27+}$ and $\mathrm{Xe}^{48+}$ have been produced by intense optical lasers ${ }^{53}$, the production of dense $\mathrm{Kr}^{34+}$ and $\mathrm{Xe}^{52+}$ plasmas has not been demonstrated yet. Following the same calculation procedure used for argon plasmas, we estimate that an optical laser with an intensity of $1.1 \times 10^{17} \mathrm{~W} \mathrm{~cm}^{-2}$ and pulse duration of about 7 ps might be needed to achieve the plasma conditions for $\mathrm{Xe}^{52+}$ shown in Fig. 2. The estimated power and energy of $88 \mathrm{TW}$ and $616 \mathrm{~J}$, respectively, are currently not available at XFEL high-energy-density (HED) endstations. However, they can be reached at current high-power laser facilities ${ }^{60}$, where intense optical laser pulses with durations from $100 \mathrm{fs}$ to $10 \mathrm{ps}$ and energies of up to $1 \mathrm{~kJ}$ are typically available, indicating the feasibility to generate dense $\mathrm{Xe}^{52+}$ ions for $\mathrm{x}$-ray lasing by using petawatt lasers in combination with XFEL facilities. Due to high thermal load ${ }^{67}$, the repetition rate of such petawatt lasers is typically below $10 \mathrm{~Hz}\left(\mathrm{ref}^{60}\right)$. However, a much higher repetition rate can be achieved when a large-scale fibre amplifier becomes available ${ }^{68}$.

Moreover, instead of using high-power optical lasers, a plasma consisting of highly charged ions can also be produced by an intense XFEL pulse ${ }^{30,69}$. The production of dense $\mathrm{H}$-like ions directly by an XFEL pulse has already been demonstrated in $\mathrm{Al}$ plasmas ${ }^{70,71}$. However, the volumes of these plasmas are too small (around 9 $\left.\mu \mathrm{m}^{2} \times 1 \mu \mathrm{m}\right)$ to obtain $\mathrm{x}$-ray lasing. Due to large absorption, it is currently inefficient to use an XFEL pulse to generate the millimeter-long dense HCI plasmas required for high-intensity XRLs. For instance, in the XRL experiment with $\mathrm{Cu}$ foils, a significant attenuation of XFEL photons was observed after a propagation of $20 \mu \mathrm{m}\left(\mathrm{ref} .{ }^{23}\right.$ ). In contrast, the use of intense optical lasers proposed here can enable the generation of dense plasmas of HCIs with lengths up to tens of millimeter or even centimeters ${ }^{24,25}$, rendering them good candidates for demonstrating hard-XRLs with saturated intensities. Furthermore, though generating dense HCIs with XFEL pulses is possible for light elements, it is challenging to control the charge-state distribution and the pump rate separately. Using a laser-produced plasma instead of generating HCIs by the XFEL pump pulse itself ${ }^{30}$ results in a more efficient scheme, with more degrees of freedom to control and optimize the output of the XRL.

Feasibility and limitations. We would like to point out that the plasma conditions presented in Fig. 2, such as temperature, density, and charge-state distributions, were obtained via steady-state atomic-physics plasma simulations, and represent a first approximation of the highly active medium ${ }^{72,73}$. Though there have been varieties of soft-x-ray lasers demonstrated from dense $\mathrm{Ne}$ - or Ni-like HCI plasmas ${ }^{26-28}$, direct experimental evidence of the plasma medium required by our lasing scheme, especially in the high- $Z$ regime ${ }^{53}$, is still missing. Theoretically, an accurate prediction of the laser conditions needed to produce such plasmas would be beneficial, but this requires the inclusion of many complex and nonlinear light-matter interactions, which goes beyond the scope of the present article. Instead, our estimations provide a first insight into the possibility of producing the required plasmas with currently available or planned intense laser facilities. These promising results motivate future experimental investigations of high-energy-density physics ${ }^{74}$ at high-power laser and XFEL facilities worldwide ${ }^{60,75-80}$, as well as further theoretical studies of laser-matter interactions incorporating particle-in-cell (PIC) simulations ${ }^{81,82}$ and XRL modeling beyond the two-level approximation ${ }^{42}$.

Summary and outlook. Our numerical simulations employing the Maxwell-Bloch equations, involving realistic parameters calculated from atomic structure theory and plasma simulations, show that the lasing scheme we put forward can be realized with currently available or planned high-power laser facilities at several XFEL endstations ${ }^{75-80}$. Such $\mathrm{x}$-ray sources will enable novel studies of coherent light-matter interactions in atomic, molecular and solid-state systems ${ }^{83}$, e.g., $\mathrm{x}$-ray quantum optics, high-resolution spectroscopy, and nonlinear x-ray scattering processes. As an example, there have been many experiments investigating the nonlinear interactions between $\mathrm{X}$-rays and matter using intense XFEL pulses ${ }^{8,38-44}$, where several states are excited and many processes are involved simultaneously. However, the XRL proposed here can be used to excite an individual state, due to the good temporal coherence and saturated intensity, and could thus complement the capabilities at broadband, incoherent XFEL sources. This includes, e.g., X-ray stimulated Raman adiabatic passage for the coherent preparation of HCIs towards generating an $\mathrm{x}$-ray frequency comb ${ }^{9}$, and resonant excitation of highly charged ions in an electron-beam ion trap for precision tests of quantum electrodynamics ${ }^{84,85}$.

Since the XRLs proposed here are seeded by spontaneous emissions from all the magnetic sublevels, similar to other XRLs, the photons are not polarized. However, one can gain control on the polarization by using a polarimeter $^{86}$, or by seeding the XRL with an external polarized $\mathrm{x}$-ray field ${ }^{87,88}$. Furthermore, a three/four-wave mixing scheme that couples the XRL with another optical/EUV laser can be investigated to tune the discrete XRL frequency in a wide range $\mathrm{e}^{89,90}$. The XRLs put forward here can also be used to perform coherent X-ray pump-probe experiments for investigations of structural relaxation processes ${ }^{91}$.

\section{Methods}

Maxwell-Bloch theory. Maxwell-Bloch theory has been successfully applied to describe the amplification and absorption of light in active media ${ }^{57}$, including the development of soft x-ray lasers in laser-produced plasmas $^{92}$. After the experimental realization of XRL pumped by an XFEL pulse, in ref. ${ }^{59}$, Weninger and Rohringer successfully applied this theory to model the evolution of the corresponding XRL. In the following, we extend this theory to also include lasing from multipole transitions, i.e., $M 1$ and $M 2$ transitions. Assuming XFEL pulses 
propagating along the $\hat{x}$ direction, the evolution of the XRL field in the slowly varying envelope approximation is given by ${ }^{57,59,92}$

$$
\frac{\partial \mathscr{A}(x, t)}{\partial t}+c \frac{\partial \mathscr{A}(x, t)}{\partial x}=i \frac{\mu_{0} \omega c^{2}}{2} \mathcal{F}(x, t),
$$

where $\mathscr{A}(x, t)$ is either the envelope of the electric field $\mathcal{E}(x, t)$ or the envelope of the magnetic field $\mathcal{B}(x, t)$, depending on the specific transition. $\mu_{0}$ is the vacuum permeability and $\omega$ is the carrier frequency of the XRL. $\mathcal{F}(x, t)$ corresponds to the polarization field induced by $\mathcal{E}(x, t)$ for $E 1$ transitions, or the magnetization field and magnetic-quadrupole field induced by $\mathcal{B}(x, t)$ for $M 1$ transitions and $M 2$ transitions, respectively.

Under the two-level approximation in He-like ions, we assume all Li-like ions are pumped into the corresponding upper lasing state of such transition by the XFEL pulse. Using $|\mathrm{e}\rangle$ and $|\mathrm{g}\rangle$ to represent the upper lasing state and the lower lasing state, respectively, the dynamics of the He-like ions are described by the Bloch equations of the density matrix

$$
\begin{gathered}
\dot{\rho}_{\mathrm{ee}}(x, t)=-\operatorname{Im}\left[\Omega^{*}(x, t) \rho_{\mathrm{eg}}(x, t)\right]+\sigma_{0} j_{\mathrm{xfel}}(x, t) \rho_{00}(x, t) \\
-\sigma_{\mathrm{e}} j_{\mathrm{xfel}}(x, t) \rho_{\mathrm{ee}}(x, t)-\Gamma \rho_{\mathrm{ee}}(x, t), \\
\dot{\rho}_{\mathrm{eg}}(x, t)=\frac{i}{2} \Omega *(x, t)\left(\rho_{\mathrm{ee}}(x, t)-\rho_{\mathrm{gg}}(x, t)\right) \\
\quad-\frac{\gamma}{2} \rho_{\mathrm{eg}}(x, t)+G(x, t), \\
\dot{\rho}_{\mathrm{gg}}(x, t)=\operatorname{Im}\left[\Omega^{*}(x, t) \rho_{\mathrm{eg}}(x, t)\right]-\sigma_{\mathrm{g}} j_{\mathrm{xfel}}(x, t) \rho_{\mathrm{gg}}(x, t) \\
+\Gamma \rho_{\mathrm{ee}}(x, t) .
\end{gathered}
$$

The carrier frequency $\omega$ is chosen to be resonant with the lasing transition $\omega_{0}$. $\rho_{\mathrm{ee}}$ and $\rho_{\mathrm{gg}}$ are the populations of $|\mathrm{e}\rangle$ and $|\mathrm{g}\rangle$, and the off-diagonal term $\rho_{\text {eg }}$ represents the coherence between the two lasing states. $\Omega(x, t)=\wp \mathcal{E}(x, t) / \hbar$ for an $E 1$ transition (or $\Omega(x, t)=m \mathcal{B}(x, t) / \hbar$ for an $M 1$ transition, and $\Omega(x, t)=k_{0} q_{y x} \mathcal{B}(x, t) / \hbar$ for an $M 2$ transition $\left.^{93}\right)$ is the time- and space-dependent Rabi frequency, with $\wp$ the electric-dipole moment, $m$ the magnetic-dipole moment, $k_{0}$ the wavenumber and $q_{y x}$ the $y x$-component of the magnetic-quadrupole tensor. For a magnetic-quadrupole transition, $k_{0} q_{y x}$ represents the effective magnetic-dipole moment coupling with the magnetic field. XFEL pumping of the $|e\rangle$ state from Li-like ions is accounted for through the second term in the right-hand side of Eq. (4), with $\rho_{00}$ being the population of Li-like ions, $j_{\text {xfel }}$ the photon flux of the XFEL pump pulse, and $\sigma_{0}$ the K-shell photoionization cross section of the pump process $\left(1 s^{2} 2 l \rightarrow 1 s 2 l\right)$. The XFEL pulse also depletes the $|\mathrm{e}\rangle$ and $|\mathrm{g}\rangle$ states, as modeled by the third term on the right-hand side of Eq. (4) and the second term on the right-hand side of Eq. (6), respectively, with $\sigma_{\mathrm{e}}$ and $\sigma_{\mathrm{g}}$ being the corresponding photoionization cross sections. In Eq. (4) and (6), $\Gamma \rho_{\mathrm{ee}}(x, t)$ describes spontaneous emission at rate $\Gamma$. In Eq. (5), the parameter

$$
\gamma=\Gamma+\Delta \omega_{\mathrm{e}-\mathrm{i}}+\left(\sigma_{\mathrm{e}}+\sigma_{\mathrm{g}}\right) j_{\mathrm{xfel}}(x, t)
$$

models the three contributions to the decay of the off-diagonal elements: $\Gamma$ is the decoherence originating from spontaneous photon emission; the second term $\Delta \omega_{\mathrm{e}-\mathrm{i}}$ accounts for the broadening from electron-ion collisions ${ }^{56}$; and the final term describes the contribution from depletion of the total population of He-like ions. To model seeding from spontaneous emission, $G(x, t)$ in Eq. (5) is a Gaussian white-noise term added phenomenologically, which satisfies $\left\langle G^{*}(x, t) G\left(x, t^{\prime}\right)\right\rangle=K(x, t) \delta\left(t-t^{\prime}\right)$. For $E 1$ transitions, one has ${ }^{92}$

$$
K(x, t)=\frac{\varepsilon_{0} \hbar \omega_{0}}{N_{\text {ion }} \wp^{2}} \frac{\Theta}{8 \pi} \frac{\gamma^{2}}{\omega_{0}^{2}} \Gamma \rho_{\mathrm{ee}}(x, t),
$$

where $\Theta \sim S_{\text {focal }} / L^{2}$ represents the solid angle in the forward direction, with $S_{\text {focal }}$ the focal size of the XFEL pulse and $L$ the length of the plasma.

The coupling between the Maxwell equations and the Bloch equations is given through the induced fields $\mathcal{F}=-2 N_{\text {ion }} d \rho_{\text {eg }}$, with $d=\wp, m, k_{0} q_{y x}$ being the effective dipole and quadrupole moments for $E 1, M 1$, and $M 2$ transitions, respectively ${ }^{57}$. Absorption of the XFEL pulse by the ions is included through the rate equations

$$
\frac{\partial j_{\mathrm{xfel}}}{\partial z}=-\sum_{\mathrm{k}} \sigma_{\mathrm{k}} \rho_{\mathrm{kk}} N_{\mathrm{ion}} j_{\mathrm{xfel}},
$$

where $\mathrm{k}$ represents any state that can be ionized by the XFEL pulse. The effect of other charge states, namely the $\mathrm{C}-, \mathrm{B}-$ and Be-like ions, is also accounted for via rate equations.

Steady-state FLYCHK plasma simulation. The generation of HCI plasmas consists of two distinct stages $^{52-54}$ : at the initial stage, it is dominated by nonlinear processes such as field-induced ionization and tunneling ionization leading to the production of lower charge states by ejection of the outermost electrons; this usually 
takes a few femtoseconds or less; after that, the ionization potential is too deep to be reached and these nonlinear processes become inefficient. Therefore, in the second stage, linear processes such as collisional ionization induced by hot electrons become dominant in the production of higher charged ions. Due to the small size of the plasma, photons emitted by spontaneous emission, radiative recombination and bremsstrahlung immediately escape the plasma, leading to a radiative loss and a decrease of the electron temperature. The role of the intense laser at this stage is to stabilize the electron temperature via reaccelerating the electrons to compensate for the radiative loss.

At a later stage, the plasma will evolve into a steady-state where linear processes such as collisional ionization, excitation, radiative recombination and spontaneous emission equilibrate with each other, and collisional-radiative atomic plasma codes such as FLYCHK can be used to simulate the corresponding plasma conditions under given electron temperature and density. Although the FLYCHK code does not include the optical laser field, the effects of the field on the HCI plasma are effectively accounted for in our FLYCHK simulation via the assumption of a stable thermal electron temperature approximated by the pondermotive scaling law in Eq. (2). Such a Maxwellian electron distribution has found to be a good approximation in previous simulations of laser-produced plasmas ${ }^{62,63}$ and is used here to show the feasibility of our scheme. Nevertheless, the optical field will modify the electron distributions, and PIC simulations ${ }^{81,82}$ might be needed to estimate the electron distributions for more accurate FLYCHK simulations.

The background radiation field in the FLYCHK simulations is neglected due to the small lateral size of the elongated plasma. This is mainly because, for a linear plasma with a $\sim 10 \mu \mathrm{m}^{2}$ cross-sectional area, photons emitted by the ions and electrons immediately escape from the plasma with negligible reabsorption. Therefore, the radiative temperature is set to be zero for all the FLYCHK simulations, i.e., no blackbody radiation field is considered. The validity of such a treatment can be confirmed from the FLYCHK simulations, where the calculated optical depth is $\sim 10^{-4}$ or smaller for photon energies beyond $10 \mathrm{eV}$.

Line broadening effects in the plasma. Doppler broadenings shown in Tables 3 and 4 are calculated as

$$
\Delta \omega_{\mathrm{D}}=\sqrt{\frac{8 \ln 2 k_{\mathrm{B}} T_{\mathrm{i}}}{m_{\mathrm{i}} c^{2}}} \omega_{0},
$$

with $k_{\mathrm{B}}$ being the Boltzmann constant, $T_{\mathrm{i}}$ the ion temperature and $m_{\mathrm{i}}$ the mass of the He-like ions. This is not significant for light ions, but it becomes dominant for heavy ions like $\mathrm{Kr}^{34+}$ and $\mathrm{Xe}^{52+}$. For the plasma conditions used in the main text, the electron-ion equilibration time is in the range of hundreds of picoseconds ${ }^{94}$. Therefore, the ion temperature is assumed to be not significantly modified during the plasma generation and the lasing process, which is around a few picoseconds or less.

The electron-impact broadening is given by ${ }^{56}$

$$
\Delta \omega_{\mathrm{e}-\mathrm{i}}=-\frac{16}{3 \overline{\mathrm{v}}_{\mathrm{e}}} \frac{N_{\mathrm{e}} \hbar^{2}}{Z_{\mathrm{i}}^{2} m_{\mathrm{e}}^{2}} \ln \Lambda\langle\boldsymbol{r} \boldsymbol{r}\rangle
$$

with $\bar{v}_{\mathrm{e}}=\sqrt{8 k_{\mathrm{B}} T_{\mathrm{e}} / \pi m_{\mathrm{e}}}$ being the average thermal velocity of the electrons in the plasma and $N_{\mathrm{e}}$ the electron density, and $Z_{\mathrm{i}}$ the charge number of the ions. $T_{\mathrm{e}}$ and $m_{\mathrm{e}}$ are the electron temperature and electron mass, respectively. $\ln \Lambda \sim 10$ is the Coulomb logarithm and $\mathbf{r r}$ is a tensor with $\mathbf{r}$ being the dipole operator of the bound electrons in the ions. Such broadening is significant only for light ions such as $\mathrm{Ne}^{8+}$ and $\mathrm{Ar}^{16+}$ with lower electron temperatures, but becomes negligible compared to $\Delta \omega_{\mathrm{D}}$ for heavy ions and higher electron temperatures.

The quadratic Stark broadening from ion-ion interaction is calculated through ${ }^{56}$

$$
\Delta \omega_{\mathrm{i}-\mathrm{i}}=\alpha \overline{F^{2}}
$$

with

$$
\begin{gathered}
\alpha=-\frac{1}{4 \pi \hbar} \sum_{k \neq j} \wp_{j k}^{2} /\left(e E_{k j}^{2}\right), \\
\overline{F^{2}}=4 Z_{\mathrm{p}}^{2} e^{2} N_{\mathrm{ion}}^{\frac{4}{3}} /\left(\pi \varepsilon_{0}\right)^{2},
\end{gathered}
$$

where $\alpha$ is the quadratic Stark coefficient which is different for each transition, and $\overline{F^{2}}$ is the mean-square electric-field strength generated by nearby perturbing ions with charge number $Z_{\mathrm{p}} \cdot \wp_{j k}$ and $E_{j k}$ are the electric-dipole moment and energy difference between the states $|j\rangle$ and $|\mathrm{k}\rangle$, respectively. Populations in different charge states $Z_{\mathrm{p}}$, as predicted from FLYCHK simulations, are also fully taken into account by using $\left\langle Z_{\mathrm{p}}^{2}\right\rangle$ in the prediction of the mean-square field $\overline{F^{2}}$. $\Delta \omega_{\mathrm{i}-\mathrm{i}}$, in general, is negligible for $N_{\mathrm{ion}}<10^{19} \mathrm{~cm}^{-3}$, but becomes large for dense ion gases.

In general, the natural broadening and electron-ion impact broadening are homogeneous for each ion, yielding a Lorentzian line profile. The Doppler broadening and ion-ion Stark broadening, on the other hand, are inhomogeneous for different ions, and result in a Gaussian profile. For systems involving both homogeneous and inhomogeneous broadenings, as it is the case here, the real spectrum has a Voigt line shape given by the convolution of the Lorentzian and Gaussian profiles, with a FWHM $\Delta \omega_{\mathrm{V}}$ approximately given by ${ }^{95}$ 


$$
\Delta \omega_{\mathrm{V}}=0.5364 \Delta \omega_{\mathrm{L}}+\sqrt{0.2166 \Delta \omega_{\mathrm{L}}^{2}+\Delta \omega_{\mathrm{G}}^{2}}
$$

Here, $\Delta \omega_{\mathrm{L}}=\Gamma+\Delta \omega_{\mathrm{e}-\mathrm{i}}$ is the FWHM of the Lorentzian function, and $\Delta \omega_{\mathrm{G}}=\Delta \omega_{\mathrm{D}}+\Delta \omega_{\mathrm{i}-\mathrm{i}}$ is the FWHM of the Gaussian function.

Numerical simulations of the lasing process accounting for the inhomogeneous broadening should take into account the distributions of the thermal velocity as well as Stark shift of the ions. This renders the simulations time consuming. However, Eq. (15) shows that

$$
\begin{aligned}
\Delta \omega_{\mathrm{V}} & \sim \Delta \omega_{\mathrm{L}}+\Delta \omega_{\mathrm{G}} \\
& =\Gamma+\Delta \omega_{\mathrm{e}-\mathrm{i}}+\Delta \omega_{\mathrm{D}}+\Delta \omega_{\mathrm{i}-\mathrm{i}}
\end{aligned}
$$

so that, for the sake of simplicity, we can approximate the parameter in Eq. (7) as

$$
\begin{aligned}
\gamma= & \Gamma+\Delta \omega_{\mathrm{e}-\mathrm{i}}+\Delta \omega_{\mathrm{D}}+\Delta \omega_{\mathrm{i}-\mathrm{i}} \\
& +\left(\sigma_{\mathrm{e}}+\sigma_{\mathrm{g}}\right) j_{\mathrm{xfel}}(x, t) .
\end{aligned}
$$

With this approximation, the distribution of the ions over different thermal velocities and Stark shifts is included in the Maxwell-Bloch equations. This simplification may lead to a maximum of $25 \%$ overestimate of the bandwidth compared to the Voigt bandwidth $\Delta \omega_{\mathrm{V}}$ in the simulations. However, this will not change our conclusions in the main text.

Furthermore, the electron density and charge-state distribution are changing during the pumping and lasing processes, leading to a time-dependent broadening effect. This effect, accounting for less than $10 \%$ of the total broadening, is neglected in our simulation.

Numerical XRL simulations. We simulate the lasing process by solving the Maxwell-Bloch equations numerically in retarded-time coordinates, with a time step of $\delta t=0.001 \tau$ and a grid size of $\delta x=c \delta t$, for 1,000 different realizations of SASE XFEL pulses. Small time-dependent changes in the broadenings due to modified charge-state distributions, densities and temperatures are neglected. Compared to $\Gamma$ and $\Delta \omega_{\mathrm{e}-\mathrm{i}}$ which are homogeneous for each ion, $\Delta \omega_{\mathrm{D}}$ and $\Delta \omega_{\mathrm{i}-\mathrm{i}}$ are inhomogeneous broadenings. Without modifying the main conclusions, they are effectively accounted for in the simulation by redefining the decoherence rate in Eq. (7) according to Eq. (17). Values of the initial populations of the states in different ions under given plasma conditions are taken from the charge-state distribution shown in Fig. 2, with negligible populations in the excited states ${ }^{51}$.

The XFEL bandwidths listed in Table 3 are chosen based on realistic parameters at XFEL facilities in operation or under construction. However, for the case of $\mathrm{Kr}^{34+}$ and $\mathrm{Xe}^{52+}$ in Table 4, the time step employed in our numerical calculations of the Maxwell-Bloch equations is $\delta t=0.95 \mathrm{fs}(\delta t=0.0001 \tau)$ for $\mathrm{Kr}^{34+}$ and $\delta t=0.34$ fs for $\mathrm{Xe}^{52+}$, respectively. This time step $\delta t$ sets the limit on the smallest coherence time, thus the largest bandwidth, we can achieve in modeling the SASE XFEL pulse. Nevertheless, since the photoionization cross sections do not change significantly within the $0.1 \%$ relative bandwidth of the XFEL peak ${ }^{47}$, given the same flux, the simulations with larger bandwidths would provide the same results as we obtained in Table 4. Therefore, when we compare the bandwidth of the XRLs and XFEL pulses, we refer to the realistic bandwidth measured at XFEL facilities and not to the values used in Table 4.

XFEL focal size. We assume that the XFEL pulse can be treated as a Gaussian beam whose transverse intensity is given by a Gaussian function. To ensure that the beam divergence does not reduce the intensity significantly after a propagation distance of $L_{c}$ shown in Tables 3 and 4, the focal depth of the XFEL beam should be comparable with the optimal plasma length $L_{c}$. Therefore, the minimum size of the XFEL focal spot for each transition shall be determined according to

$$
S_{\text {focal }}=\frac{L_{\mathrm{c}}}{2} \lambda_{\text {xfel }} \text {. }
$$

The calculated values, in units of $\mu m^{2}$, are 1.4, $0.5,0.05,10.5$ and 0.13 for the systems listed in Tables 3 and 4 , respectively.

Another factor determining the focal size is the photon number. The maximum values of photons per pulse at given XFEL photon energies can be estimated from Fig. 19 in ref. ${ }^{4}$, which are around $2 \times 10^{14}, 5 \times 10^{13}, 4 \times 10^{12}$ and $1 \times 10^{12}$ for XFEL photon energies of 1.2, 4.1, 17 and $40 \mathrm{keV}$, respectively. Given the photon flux and pulse duration listed in Tables 3 and 4, one obtains another bound for the XFEL focal size via

$$
S_{\text {focal }}=\frac{\text { photons per pulse }}{(\text { photon flux }) \cdot(\text { pulse duration })},
$$

which gives the values of $85,1.8,12,0.1$ and $0.02 \mu \mathrm{m}^{2}$ for the corresponding systems, respectively. Comparing the two bounds, we choose to use the focal sizes of $1.5,1.0,0.1,0.1$ and $0.13 \mu \mathrm{m}^{2}$, respectively, as listed in Tables 3 and 4 .

Laser parameters required for the generation of Ar plasma. In order to show how the plasmas discussed in this paper can be produced with current laser facilities, we provide a conservative estimation of the laser conditions necessary to generate, e.g., a plasma consisting of highly charged Ar ions, for lasing from the ${ }^{3} P_{1} \rightarrow{ }^{1} S_{0}$ 
transition in $\mathrm{Ar}^{16+}$. This requires one to consider the time and energy needed to produce the plasma from a neutral gas and, at the same time, the time and total energy required for the plasma to evolve into a steady-state characterized by the corresponding charge-state distribution (red line) shown in Fig. 2.

First, the energy per electron necessary to generate such plasma can be conservatively estimated as the sum of the kinetic energy of the electron gas $\left(T_{\mathrm{e}}=250 \mathrm{eV}\right)$ and the average ionization energy $W_{0}=366 \mathrm{eV}$ per electron. The latter is determined by the mean value of first, second, ..., sixteenth ionization energies, and calculated based on the ionization energy data from the NIST Atomic Spectra Database ${ }^{96}$. Thus, the total energy deposited in the plasma can be approximated as

$$
W \approx\left(T_{\mathrm{e}}+W_{0}\right) N_{\mathrm{e}} S L
$$

$N_{e}$ is the electron density, while $S$ and $L$ are the cross-sectional area and the length of the plasma, respectively. For simplicity, we assume that all the ions in the plasma are He-like ions such that $N_{\mathrm{e}} \approx 16 N_{\mathrm{ion}}$. Supposing that an intense optical laser with a line focus of $10 \mu \mathrm{m} \times 4 \mathrm{~mm}$ (given the optimal length of $3.3 \mathrm{~mm}$ shown in Table 3) illuminates the Ar gas of a thickness of $1 \mu \mathrm{m}$, we estimate $W \approx 1.6 \mathrm{~mJ}$. With an absorption rate of the laser pulse of $10 \%$ (ref. ${ }^{97}$ ), this requires a laser of an energy of $16 \mathrm{~mJ}$. Based on Eq. (2), obtaining such an Ar plasma from a laser of a wavelength of $800 \mathrm{~nm}$ requires a laser intensity of about $1.1 \times 10^{15} \mathrm{~W} \mathrm{~cm}^{-2}$. With a $10 \mu \mathrm{m} \times 4 \mathrm{~mm}$ linear geometry, the power needed for such an experiment is estimated as $0.44 \mathrm{TW}$. For an energy of $16 \mathrm{~mJ}$, this implies a pulse duration of $\sim 36 \mathrm{fs}$.

However, in order to let the plasma evolve into a steady state characterized by the corresponding charge-state distribution (red line in Fig. 2), the optical laser is required also to compensate for the energy loss from processes such as recombination and to prevent the electron gas from cooling down ${ }^{73,98}$. Therefore, the pulse duration of the optical laser should be long enough to cover the whole plasma generation, equilibration and lasing processes. For the plasma conditions shown in Fig. 2, the collisional-ionization times obtained from the FLYCHK simulations are of the order of a few picoseconds or fractions thereof. Together with the subsequent 1 ps lasing process shown in Fig. $4 \mathrm{a}$, the whole process lasts approximately 2 ps for the corresponding Ar plasma. Given the $0.44 \mathrm{TW}$ power calculated above, such pulse duration corresponds to a total energy of $0.88 \mathrm{~J}$, which is achievable at currently existing high-power laser facilities ${ }^{60,65,66}$.

\section{Data availability}

The data that support the findings of this study are available from the corresponding author upon reasonable request.

\section{Code availability}

The codes used to generate the results reported in this study are available from the corresponding author upon reasonable request.

Received: 30 October 2019; Accepted: 1 May 2020;

Published online: 10 June 2020

\section{References}

1. Pellegrini, C., Marinelli, A. \& Reiche, S. The physics of x-ray free-electron lasers. Rev. Mod. Phys. 88, 015006 (2016).

2. Berrah, N. et al. Non-linear processes in the interaction of atoms and molecules with intense EUV and X-ray fields from SASE free electron lasers (FELs). J. Mod. Opt. 57, 1015 (2010).

3. Bostedt, C. et al. Linac Coherent Light Source: The first five years. Rev. Mod. Phys. 88, 015007 (2016).

4. Seddon, E. et al. Short-wavelength free-electron laser sources and science: a review. Rep. Prog. Phys. 80, 115901 (2017).

5. Adams, B. W. et al. X-ray quantum optics. J. Mod. Opt. 60, 2 (2013).

6. Liao W.-T. Coherent control of nuclei and $x$-rays. (Springer, 2014).

7. Heeg, K. P. et al. Spectral narrowing of $\mathrm{x}$-ray pulses for precision spectroscopy with nuclear resonances. Science 357,375 (2017).

8. Weninger, C. et al. Stimulated electronic X-ray Raman scattering. Phys. Rev. Lett. 111, 233902 (2013).

9. Cavaletto, S. M. et al. Broadband high-resolution x-ray frequency combs. Nat. Photonics 8, 520 (2014).

10. Shakhmuratov, R. N., Vagizov, F. G., Scully, M. O. \& Kocharovskaya, O. Application of the low-finesse $\gamma$-ray frequency comb for high-resolution spectroscopy. Phys. Rev. A 94, 043849 (2016).

11. Glenzer, S. H. \& Redmer, R. X-ray Thomson scattering in high energy density plasmas. Rev. Mod. Phys. 81, 1625 (2009).

12. McBride, E. E. et al. Setup for meV-resolution inelastic X-ray scattering measurements and X-ray diffraction at the Matter in Extreme Conditions endstation at the Linac Coherent Light Source. Rev. Sci. Instrum. 89, 10F104 (2018).

13. Sutton, M. et al. Observation of speckle by diffraction with coherent X-rays. Nature 352, 608 (1991).

14. Shpyrko, O. G. X-ray photon correlation spectroscopy J. Synchrotron Radiat. 21, 1057 (2014).

15. Roseker, W. Development of a hard X-ray delay line for X-ray photon correlation spectroscopy and jitter-free pump-probe experiments at X-ray free-electron laser sources. J. Synchrotron Radiat. 18, 481 (2011).

16. Lambert, G. et al. Injection of harmonics generated in gas in a free-electron laser providing intense and coherent extremeultraviolet light. Nat. Phys. 4, 296 (2008).

17. Amann, J. et al. Demonstration of self-seeding in a hardx-ray free-electron laser. Nat. Photonics 6, 693 (2012).

18. Allaria, E. et al. Highly coherent and stable pulses from the FERMI seeded free-electron laser in the extreme ultraviolet. Nat. Photonics 6, 699 (2012).

19. Ratner, D. et al. Experimental demonstration of a soft xray self-seeded free-electron laser. Phys. Rev. Lett. 114, 054801 (2015).

20. Huang, S. et al. Generating single-spike Hard x-ray pulses with nonlinear bunch compression in free-electron lasers. Phys. Rev. Lett. 119, 154801 (2017).

21. Kim, K. J., Shvyd'ko, Y. \& Reiche, S. A proposal for an xray free-electron laser oscillator with an energy-recovery linac. Phys. Rev. Lett. 100, 244802 (2008).

22. Rohringer, N. et al. Atomic inner-shell x-ray laser at 1.46 nanometres pumped by an x-ray free-electron laser. Nature 481 , 488 (2012).

23. Yoneda, H. et al. Atomic inner-shell laser at 1.5-angström wavelength pumped by an X-ray free-electron laser. Nature 524, 446 (2015). 
24. Matthews, D. L. et al. Demonstration of a soft x-ray amplifier. Phys. Rev. Lett. 54, 110 (1985).

25. Kapteyn, H. C., Lee, R. W. \& Falcone, R. W. Observation of a short-wavelength laser pumped by Auger decay. Phys. Rev. Lett. 57, 2939 (1986).

26. Rocca, J. J. Table-top soft x-ray lasers. Rev. Sci. Instrum. 70, 3799 (1999).

27. Daido, H. Review of soft $\mathrm{x}$-ray laser researches and developments. Rep. Prog. Phys. 65, 1513 (2002).

28. Suckewer, S. \& Jaegle, P. X-Ray laser: past, present, and future. Laser Phys. Lett. 6, 411 (2009).

29. Duguay, M. \& Rentzepis, P. Some approaches to vacuum UV and x-ray lasers. Appl. Phys. Lett. 10, 350 (1967).

30. Rohringer, N. \& London, R. Atomic inner-shell X-ray laser pumped by an X-ray free-electron laser. Phys. Rev. A 80, 013809 (2009).

31. Röhlsberger, R., Schlage, K., Sahoo, B., Couet, S. \& Rüffer, R. Collective Lamb shift in single-photon superradiance. Science 328, 1248 (2010).

32. Röhlsberger, R., Wille, H., Schlage, K. \& Sahoo, B. Electromagnetically induced transparency with resonant nuclei in a cavity. Nature 482, 199 (2012).

33. Vagizov, F., Antonov, V., Radeonychev, Y., Shakhmuratov, R. \& Kocharovskaya, O. Coherent control of the waveforms of recoilless $\gamma$-ray photons. Nature 508, 80 (2014).

34. Picón, A., Mompart, J. \& Southworth, S. H. Stimulated Raman adiabatic passage with two-color x-ray pulses. New J. Phys. 17, $083038(2015)$

35. Prince, K. C. et al. Coherent control with a shortwavelength free-electron laser. Nat. Photonics 10, 176 (2016)

36. Haber, J. et al. Rabi oscillations of X-ray radiation between two nuclear ensembles. Nat. Photonics 11, 720 (2017).

37. Wang, G.-Y. \& Liao, W.-T. Generation of Short HardX-Ray Pulses of Tailored Duration Using a Mssbauer Source. Phys. Rev. Applied 10, 014003 (2018).

38. Kanter, E. P. et al. Unveiling and driving hidden resonances with high-fluence, high-intensity $\mathrm{x}$-ray pulses. Phys. Rev. Lett. 107, $233001(2011)$

39. Doumy, G. et al. Nonlinear atomic response to intense ultrashort x rays. Phys. Rev. Lett. 106, 083002 (2011).

40. Beye, M. et al. Stimulated X-ray emission for materials science. Nature 501, 191 (2013).

41. Kimberg, V. \& Rohringer, N. Amplified x-ray emission from core-ionized diatomic molecules. Phys. Rev. Lett. 110, 043901 (2013).

42. Kroll, T. et al. Stimulated $\mathrm{x}$-ray emission spectroscopy in transition metal complexes. Phys. Rev. Lett. 120, 133203 (2018).

43. Tamasaku, K. et al. X-ray two-photon absorption competing against single and sequential multiphoton processes. Nat. Photonics 8, 313 (2014).

44. Shwartz, S. et al. X-ray second harmonic generation. Phys. Rev. Lett. 112, 163901 (2014).

45. Bernitt, S. et al. An unexpectedly low oscillator strength as the origin of the FeXVII emission problem. Nature 492, 225 (2012).

46. Oreshkina, N. S., Cavaletto, S. M., Keitel, C. H. \& Harman, Z. Astrophysical line diagnosis requires nonlinear dynamical atomic modeling. Phys. Rev. Lett. 113, 143001 (2014).

47. LANL Atomic Physics Codes, http://aphysics2. lanl.gov/cgi-bin/ION/runlanl08f.pl.

48. Stewart, J. C. \& Pyatt, K. D. Jr. Lowering of ionization potentials in plasmas. Astrophys. J. 144, 1203 (1966).

49. Ditmire, T., Donnelly, T., Rubenchik, A. M., Falcone, R. W. \& Perry, M. D. Interaction of intense laser pulses with atomic clusters. Phys. Rev. A 53, 3379 (1996).

50. Krainov, V. \& Smirnov, M. Cluster beams in the superintense femtosecond laser pulse. Phys. Rep. 370, 237 (2002),

51. Chung, H. K., Chen, M., Morgan, W., Ralchenko, Y. \& Lee, R. FLYCHK: Generalized population kinetics and spectral model for rapid spectroscopic analysis for all elements. High Energy Density Phys. 1, 3 (2005).

52. Murnane, M. M., Kapteyn, H. C., Rosen, M. D. \& Falcone, R. W. Ultrafast X-ray pulses from laser-produced plasmas. Science 251, 531 (1991).

53. McPherson, A., Thompson, B. D., Borisov, A. B., Boyer, K. \& Rhodes, C. K. Multiphoton-induced X-ray emission at 4-5 keV from Xe atoms with multiple core vacancies. Nature 370, 631 (1994).

54. Reich, C., Gibbon, P., Uschmann, I. \& Förster, E. Yield optimization and time structure of femtosecond laser plasma K $\alpha$ sources. Phys. Rev. Lett. 84, 4846 (2000)

55. Dyall, K., Grant, I., Johnson, C., Parpia, F. \& Plummer, E. GRASP: A general-purpose relativistic atomic structure program. Comput. Phys. Commun. 55, 425 (1989).

56. Griem, H. R. Spectral line broadening in plasmas. (Acadamic, New York, 1974).

57. Scully, M. O. \& Zubairy, M. S. Quantum optics. (Cambridge University Press, Cambridge, England, 1997).

58. Cavaletto, S. M. et al. Resonance fluorescence in ultrafast and intense x-ray free-electron-laser pulses. Phys. Rev. A 86, 033402 (2012).

59. Weninger, C. \& Rohringer, N. Transient-gain photoionization x-ray laser. Phys, Rev. A 90, 063828 (2014).

60. Danson, C., Hillier, D., Hopps, N. \& Neely, D. Petawatt class lasers worldwide. High Power Laser Sci. Eng. 3, E3 (2015).

61. Als-Nielsen, J. \& McMorrow, D. Elements of modern Xray physics. (John Wiley \& Sons, Cambridge, England, 2011).

62. Tanimoto, T. et al. Measurements of fast electron scaling generated by petawatt laser systems. Phys. Plasmas 16, 062703 (2009)

63. Nilson, P. M. et al. Time-resolved measurements of hotelectron equilibration dynamics in high-intensity laser interactions with thin-foil solid targets. Phys. Rev. Lett. 108, 085002 (2012).

64. Caillaud, T. et al. Experimental study of K-shell X-ray emission from argon clusters irradiated by an ultraintense laser pulse. Nucl. Instrum. Methods Phys. Res.B 205, 329 (2003).

65. Reagan, B. A. et al. Scaling diode-pumped, high energy picosecond lasers to kilowatt average powers. High Pow. Las. Sci. Eng. 6, e11 (2018).

66. Calendron, A. L. et al. Laser system design for tabletop X-ray light source. High Pow. Las. Sci. Eng. 6, e12 (2018).

67. Brocklesby, W. S. Progress in high average power ultrafast lasers. Eur. Phys. J. Special Topics 224, 2529 (2015).

68. Mourou, G., Brocklesby, B., Tajima, T. \& Limpert, J. The future is fibre accelerators. Nat. Photonics 7, 258 (2013).

69. Kumagai, Y. et al. Following the birth of a nanoplasma produced by an ultrashort hard-X-ray laser in xenon clusters. Phys. Rev. X 8, 031034 (2018).

70. Vinko, S. M. et al. Creation and diagnosis of a soliddensity plasma with an X-ray free-electron laser. Nature 482, 59 (2012).

71. Ciricosta, O. et al. Direct measurements of the ionization potential depression in a dense plasma. Phys. Rev. Lett. 109, 065002 (2012).

72. Hansen, S. B. et al. Review of the 10th Non-LTE code comparison workshop. High Energy Density Phys. 35, 100693 (2020).

73. Bastiani-Ceccotti, S. et al. Analysis of the X-ray and time-resolved XUV emission of laser produced Xe and $\mathrm{Kr}$ plasmas. High Energy Density Phys. 3, 20 (2007)

74. Drake, R. P. Introduction to high-energy-density physics. (Springer, Cham, 2018).

75. Zhu, Z. Y. et al. SCLF: An 8-GeV CW SCRF linacbased X-ray FEL facility in Shanghai. In Proceedings of FEL2017, Santa Fe, NM, USA, (2017).

76. Nagler, B. et al. The matter in extreme conditions instrument at the Linac coherent light source. J. Synchrotron Rad. 22, 520 (2015).

77. Glenzer, S. H. et al. Matter under extreme conditions experiments at the Linac Coherent Light Source. J. Phys. B: At. Mol. Opt. Phys. 49, 092001 (2016)

78. Nakatsutsumi, M. et al. Technical Design Report: Scientific Instrument High Energy Density Physics (HED) (European XFEL $\mathrm{GmbH}, 2014)$. 
79. Nakatsutsumi, M. et al. Femtosecond laser-generated high-energy-density states studied by x-ray FELs. Plasma Phys. Contrib. Fusion 59, 014028 (2017).

80. Yabuuchi, T. et al. An experimental platform using highpower, high-intensity optical lasers with the hard X-ray free-electron laser at SACLA. J. Synchrotron Rad. 26, 585 (2019).

81. Arber, T. D. et al. Contemporary particle-in-cell approach to laser-plasma modelling, Plasma Phys. Contrib. Fusion 57, 113001 (2015).

82. Wilks, S. C., Kruer, W. L., Tabak, M. \& Langdon, A. B. Absorption of ultra-intense laser pulses. Phys. Rev. Lett. 69, 1383 (1992).

83. Young, L. et al. Roadmap of ultrafast x-ray atomic and molecular physics. J. Phys. B: At. Mol. Opt. Phys. 51, 032003 (2018).

84. Schmöger, L. et al. Coulomb crystallization of highly charged ions. Science 347, 1233 (2015).

85. Rudolph, J. K. et al. X-ray resonant photoexcitation: Linewidths and energies of $\mathrm{K} \alpha$ transitions in highly charged Fe ions. Phys. Rev. Lett. 111, 103002 (2013).

86. Marx, B. et al. High-precision x-ray polarimetry. Phys Rev. Lett. 110, 254801 (2013).

87. Zeitoun Ph. et al. A high-intensity highly coherent soft X-ray femtosecond laser seeded by a high harmonic beam. Nature 431, 426 (2004).

88. Wang, Y. et al. Phase-coherent, injection-seeded, tabletop soft-X-ray lasers at $18.9 \mathrm{~nm}$ and $13.9 \mathrm{~nm}$. Nat. Photonics 2, 94 (2008).

89. Glover, T. E. et al. X-ray and optical wave mixing. Nature 488, 603 (2012).

90. Bencivenga, F. et al. Four-wave mixing experiments with extreme ultraviolet transient gratings. Nature 520, 205 (2015).

91. Ruta, B. et al. Atomic-scale relaxation dynamics and aging in a metallic glass probed by $\mathrm{x}$-ray photon correlation spectroscopy. Phys. Rev. Lett. 109, 165701 (2012).

92. Larroche, O. et al. Maxwell-Bloch modeling of x-raylaser-signal buildup in single-and double-pass configurations. Phys. Rev. A 62, $043815(2000)$

93. Pálffy, A., Evers, J. \& Keitel, C. H. Electric-dipoleforbidden nuclear transitions driven by super-intense laser fields. Phys. Rev. C 77, 044602 (2008).

94. Spitzer, L. Physics of fully ionized gases. (Interscience, New York, 1967).

95. Olivero, J. \& Longbothum, R. Empirical fits to the Voigt line width: A brief review. J. Quant. Spectrosc. Radiat. Transf. 17, 233 (1961).

96. NIST Atomic Spectra Database (ver. 5.5.5), https://physics.nist.gov/asd.

97. Ping, Y. et al. Absorption of short laser pulses on solid targets in the ultrarelativistic regime. Phys. Rev. Lett. 100, 085004 (2008).

98. Rupp, D. et al. Recombination-enhanced surface expansion of clusters in intense soft X-ray laser pulses. Phys. Rev. Lett. 117, 153401 (2016).

99. Lyu, C. Narrow-band hard-X-ray lasing. Ph.D. Thesis, Heidelberg University, Germany, http://archiv.ub.uni-heidelberg.de/ volltextserver/24850/ (2018).

\section{Acknowledgements}

The authors thank S. Tang, Y. Wu, J. Gunst, F. Mackenroth, and M. Tamburini for fruitful discussions. This article comprises parts of the Ph.D. thesis work of C. Lyu.

\section{Author contributions}

C.L. developed the mathematical model, performed the analytical calculations and numerical simulations, and wrote the manuscript. S.M.C. and Z.H. conceived the underlying scheme. C.L., S.M.C., C.H.K., and Z.H. contributed to the development of ideas, discussion of the technical aspects and results, and preparation of the manuscript.

\section{Competing interests}

The authors declare no competing interests.

\section{Additional information}

Supplementary information is available for this paper at https://doi.org/10.1038/s41598-020-65477-0.

Correspondence and requests for materials should be addressed to S.M.C.

Reprints and permissions information is available at www.nature.com/reprints.

Publisher's note Springer Nature remains neutral with regard to jurisdictional claims in published maps and institutional affiliations.

(1) Open Access This article is licensed under a Creative Commons Attribution 4.0 International License, which permits use, sharing, adaptation, distribution and reproduction in any medium or format, as long as you give appropriate credit to the original author(s) and the source, provide a link to the Creative Commons license, and indicate if changes were made. The images or other third party material in this article are included in the article's Creative Commons license, unless indicated otherwise in a credit line to the material. If material is not included in the article's Creative Commons license and your intended use is not permitted by statutory regulation or exceeds the permitted use, you will need to obtain permission directly from the copyright holder. To view a copy of this license, visit http://creativecommons.org/licenses/by/4.0/.

(C) The Author(s) 2020 